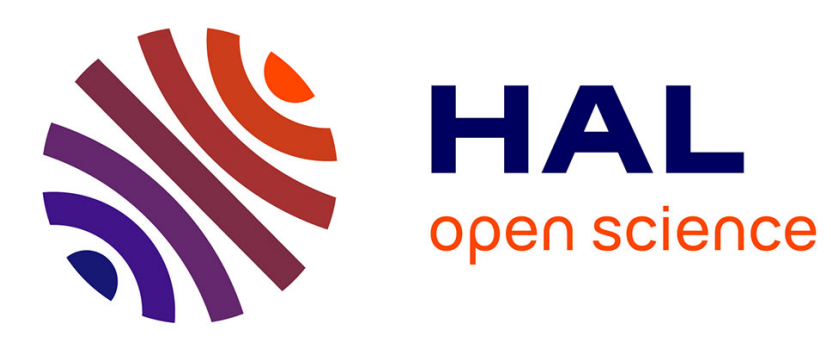

\title{
Modélisation causale pour la commande auto adaptée de machines alternatives triphasées en mode dégradé
}

\author{
Yvan Crevits, Xavier Kestelyn, Betty Lemaire-Semail, Eric Semail
}

\section{To cite this version:}

Yvan Crevits, Xavier Kestelyn, Betty Lemaire-Semail, Eric Semail. Modélisation causale pour la commande auto adaptée de machines alternatives triphasées en mode dégradé. European Journal of Electrical Engineering, 2010, 13 (6), pp.345-384. 10.3166/ejee.13.345-384 . hal-00817721

\section{HAL Id: hal-00817721 \\ https://hal.science/hal-00817721}

Submitted on 28 Jan 2014

HAL is a multi-disciplinary open access archive for the deposit and dissemination of scientific research documents, whether they are published or not. The documents may come from teaching and research institutions in France or abroad, or from public or private research centers.
L'archive ouverte pluridisciplinaire HAL, est destinée au dépôt et à la diffusion de documents scientifiques de niveau recherche, publiés ou non, émanant des établissements d'enseignement et de recherche français ou étrangers, des laboratoires publics ou privés. 


\title{
Modélisation causale pour la commande auto adaptée de machines alternatives triphasées en mode dégradé
}

\author{
Yvan Crévits* — Xavier Kestelyn* _ Betty Lemaire-Semail** \\ Éric Semail*
}

Laboratoire d'Électrotechnique et d'Électronique de Puissance de Lille

* École Nationale Supérieure d'Art Et Métiers

8, Boulevard Louis XIV, 59046 Lille, France

E-Mail : prenom.nom@lille.ensam.fr (sans accents)

** Polytech'Lille

Avenue Paul Langevin - 59655 Villeneuve d'Ascq Cedex - France

E-Mail : Betty.Semail@polytech-lille.fr

URL: http://l2ep.univ-lille1.fr

RÉSUMÉ. Cet article établit un modèle causal unique pour les fonctionnements normal et dégradé d'un entraînement électromécanique à phases non couplées en s'appuyant sur un formalisme vectoriel et une Représentation Energétique Macroscopique (REM). L'inversion systématique du modèle permet de déduire une architecture de commande dans laquelle des correcteurs spécifiques sont utilisés pour assurer les deux modes de fonctionnement. Les validations en simulation, l'examen des performances en mode dégradé par rapport au mode normal et plusieurs validations expérimentales mettent en évidence la validité de la commande établie. Enfin, la méthode développée dans l'article pour les machines triphasées est adaptable aux machines polyphasées pour mettre en évidence la généralité de la méthode.

MOTS-CLÉS : machines polyphasées, machines alternatives triphasées, filtrage de couple, commande auto adaptée, entraînements électromécaniques tolérants aux pannes.

ABSTRACT. This paper deals with a unique causal model in normal and open-circuited phase operation of a electromechanical drive with independent supplied phases. This overall degraded system model is based on a vectorial formalism and the Energetic Macroscopic Representation (EMR). The systematic inversion of the model leads to a control structure using a dedicated controller available in the two operating modes. The simulating validation, the performances verifications in open-circuited mode versus normal mode and the experimental validations establish the validity of the control. Finally, this tree-phase method is adaptable to polyphase machines to bring to light the generality of the method.

KEYWORDS: multiphase machines, alternative machines, torque filtering, auto adaptable control, fault tolerant drives. 


\section{Introduction}

L'étude en fonctionnement normal des entraînements électriques polyphasés employés dans les systèmes industriels fait l'objet de nombreux travaux (Figueroa $e t$ al., 2003 ; Figueroa et al., 2006 ; Levi et al., 2007). Lorsque l'alimentation de la machine est altérée par l'ouverture accidentelle de l'une des phases, la marche du système n'est alors plus satisfaisante. Si aucune mesure n'est prise, cela peut occasionner des conséquences défavorables : l'arrêt du système, des détériorations graves, voire la destruction de l'entraînement. Le fonctionnement peut aussi se poursuivre, mais dans des conditions très défavorables, accompagnées d'oscillations de couple. Ces dernières n'occasionnent bien souvent que des désagréments, comme des vibrations audibles dans le cas des éoliennes. Mais pour certaines applications sensibles, elles peuvent être nocives pour les constituants mécaniques, comme les paliers pour les applications à vitesse élevée, voire inadéquates, comme la perte de la furtivité de la motorisation des navires militaires. La dégradation peut même devenir critique si l'on considère que la sûreté de fonctionnement est primordiale : continuité de service dans les systèmes embarqués, maintien de paramètres de production dans l'industrie chimique ou nucléaire, etc. (Welchko et al., 2004).

De ce fait, le mode dégradé qui découle du défaut d'alimentation revêt une grande importance et l'étude de son impact sur le fonctionnement de la machine apparaît essentiel (Kestelyn, 2003). Des études ont donc été menées pour prolonger pendant une certaine durée, même limitée, le fonctionnement de l'ensemble, tout en garantissant un niveau de performances satisfaisant, ainsi que la sécurité des personnes et des équipements (Wallmark et al., 2007).

\subsection{Les machines polyphasées}

Les machines polyphasées sont caractérisées par un nombre de phases au moins supérieur à trois. Ces machines n'ont pas suscité un intérêt immédiat après leur apparition (Levi et al., 2007) et leur usage s'est plus particulièrement répandu dans deux domaines :

- L'amélioration de la sûreté de fonctionnement des motorisations électriques,

- Le fractionnement de la puissance dans les enroulements quand les puissances s'échelonnent de quelques centaines de kilowatts à plusieurs dizaines de mégawatts.

Les études du début des années 80 ont porté sur l'amélioration de la fiabilité des machines asynchrones reposant sur le principe de redondance parallèle procuré par l'augmentation du nombre de phases statoriques (Jahns, 1980). Dans ce cas, l'essentiel des études porte sur la dégradation du couple provoquée par la perte d'une ou plusieurs phases. 
Cette redondance est aussi très appréciée dans la propulsion de navires électriques. Pour les machines à double étoile par exemple, $50 \%$ de la puissance peut être préservée en cas de défaut (Filliau et al., D5620), ce qui assure la disponibilité de la motorisation.

L'autre point concerne le fractionnement de la puissance par phase nécessaire dans la motorisation des navires électriques (Filliau et al., D5610). C'est surtout le cas lorsque la puissance nominale de la machine augmente. Le fractionnement de la puissance sur plus de trois phases permet de limiter les contraintes par enroulement (Semail et al., 2003), ce qui occasionne :

- La limitation des courants afin d'éviter l'association de composants de commutation en parallèle pour constituer un interrupteur statique ;

- La limitation de la tension par phase pour éviter l'usage de convertisseurs multi niveaux ;

- La réduction naturelle des ondulations de couple et leur rejet dans le domaine des fréquences élevées.

Cette vision est appliquée aux machines polyphasées utilisées dans la propulsion électrique maritime. Jusqu'à $2 \mathrm{MW}$, les machines sont triphasées synchrones ou asynchrones. Au-delà, et jusqu'à $20 \mathrm{MW}$, l'augmentation du nombre de phases utilise des technologies de bobinages triphasés connues ou maîtrisées : 2 étoiles électriquement indépendantes déphasées de $30^{\circ}, 3$ étoiles déphasées de $20^{\circ}$, etc. D'autres machines n'utilisent pas une «base triphasée » : ce sont des machines à 4 , 5,7 phases ou plus. On rencontre même des machines combinant les deux méthodes : 2 fois 13 phases (Framatome/Jeumont) ou 3 fois 5 phases (Alstom).

Depuis les années 80 , la modélisation et la commande des machines polyphasées s'est accompagnée d'étapes décisives quelque soit l'application envisagée ou l'objectif poursuivi.

Tout d'abord, l'augmentation de la dynamique des systèmes a nécessité d'alimenter la machine par des onduleurs de tension pleine onde en lieu et place des onduleurs de courant. Dans ce cas, les harmoniques de tension génèrent des courants «parasites » qui ne produisent pas de couple. Ces harmoniques sont mis en évidence en utilisant les composantes symétriques « polyphasées » appliquées à des tensions périodiques non sinusoïdales (Klingshirn, 1983). L'étude montre aussi que les courants parasites sont organisés par groupes d'harmoniques que l'auteur élimine à l'aide de filtres placés en série avec les enroulements (Klingshirn, 1985). Mais cette recherche de solution ne peut être effectuée qu'en régime permanent.

Dans les années 90, les machines polyphasées font l'objet d'un intérêt croissant et la réflexion se porte sur la conception de machines visant à améliorer leurs performances lorsqu'elles sont alimentées par des onduleurs de tension pleine onde (Toliyat et al., 1991). Dans ce cas, l'usage d'enroulements concentrés est plus adapté à la forme rectangulaire des tensions. C'est aussi l'occasion d'établir le 
modèle de la machine aussi bien en régime permanent qu'en régime transitoire. Deux groupes de machines sont ainsi mis en évidence : celles de type 1 (3, 6 phases) et celles de type 2 (5, 7 et 9 phases). Avec une machine à 5 phases, Toliyat exploite l'harmonique de rang 3 de la f.e.m. pour assurer un accroissement de couple de $10 \%$. À ce stade, la mise en équation des machines polyphasées en vue de réaliser leur commande utilise un lot d'équations différentielles fortement couplées auxquelles sont appliquées des transformations mathématiques propres aux machines étudiées. La formalisation par vecteur d'espace (Zhao et al., 1995) apporte l'idée de décomposition des relations dans des espaces orthogonaux.

Au début des années 2000, (Semail, 2000) met en place le formalisme vectoriel généralisé pour décrire les machines et les convertisseurs. Il décompose les machines polyphasées dans des espaces orthogonaux. Chacun d'entre eux est associé à une machine fictive. Par ce biais, les machines polyphasées entrent dans le cadre des systèmes multimachines (SMM) (Semail et al., 2005). La représentation énergétique macroscopique (REM) complète cette vision des systèmes à l'aide d'un outil visuel et synthétique (Bouscayrol, 2000).

\subsection{Machines polyphasées en mode dégradé}

Dans un cadre général, la dégradation peut apparaitre au niveau des organes mécaniques (accouplements, roulements, etc.), aspects écartés de la présente étude. Sur le plan électrique, la dégradation de l'alimentation de la machine peut toucher le convertisseur au niveau des bras de l'onduleur, les composants de la machine (enroulements, barres ou aimants rotoriques, etc.) ou les liaisons électriques entre les deux éléments de la chaîne d'énergie. En cas de dégradation, la phase détériorée doit être isolée pour éviter la propagation du défaut aux autres phases. Par conséquent, quelque soit la dégradation, la phase perturbée est caractérisée par l'annulation du courant correspondant (Welchko et al., 2002 ; Martin et al., 2007).

La correction de la dégradation tient compte du nombre de phases dont dispose la machine. Quand leur nombre est important, essentiellement au-delà de neuf, la perte d'une phase provoque une dégradation sur le couple qui peut être tolérée (Williamson et al., 2004). Effectivement, l'ondulation de couple est d'autant moins importante que le nombre de phases s'accroît. Dans ce cas, aucun traitement de la dégradation n'est effectué si le « taux d'ondulation » du couple reste acceptable avec des courants limités dans les phases saines.

Pour les machines de moins de neuf phases, une stratégie de remédiation est envisagée pour gérer au mieux le transfert de puissance avec les phases restantes tout en limitant la surcharge occasionnée. Ce principe souvent repris dans la littérature reconstruit le modèle de la machine rendue dissymétrique par la dégradation de l'alimentation. Pour réduire l'ondulation de couple, le traitement de l'ouverture d'une ou plusieurs phases suit alors deux stratégies principales : 
- la modification du courant d'une phase encore saine pour chaque phase ouverte (Martin et al., 2000) ;

- la modifications des courants dans toutes les phases encore saines (Fu, 1994 ; Toliyat, 1998).

La commande déduite du modèle en mode dégradé permet de maintenir le fonctionnement dans les conditions les plus satisfaisantes possibles. En travaillant dans la base naturelle, la correction des courants restants dans les phases saines nécessite l'évaluation analytique des nouvelles références pour toutes les configurations de défauts possibles. Ceci a fait l'objet de divers exemples représentatifs (Zhao et al., 1996 ; Levi et al., 2007 ; Semai1, 2007).

Avec le formalisme vectoriel généralisé des machines polyphasées (Semail, 2000), le modèle de la machine est décomposé en machines fictives ; la dégradation est alors analysée dans ces machines, ce qui permet d'y calculer les nouvelles références permettant d'asservir les courants de façon plus aisée. Cette méthode a été utilisée dans (Crévits et al, 2006 ; Locment et al., 2006).

Pour conclure cet état de l'art, on peut noter que toutes ces notions ont fait l'objet de travaux au sein du groupe de recherche (GdR) «Sûreté et Disponibilité des Systèmes Électrotechniques 》(SDSE) dans la thématique « Systèmes Multimachines Multiconvertisseurs » (SMM) entre 1998 et 2001 puis au sein de la thématique «systèmes » du GdR « Maîtrise de l'Énergie Électrique : du matériau au système » (ME2MS) de 2002 à 2005.

\subsection{Démarche suivie, objectif et organisation}

Même s'il est possible d'étendre notre propos à un nombre de phases plus élevé sans perte de généralité, notre étude concerne ici les machines alternatives triphasées, synchrones ou asynchrones. Pour profiter de tous les degrés de liberté de cette machine, son alimentation électrique est découplée (ni couplage étoile, ni triangle ou autre). L'objectif de l'étude est de rechercher une méthode permettant de poursuivre un fonctionnement en mode dégradé d'alimentation (ouverture d'une phase statorique) tout en conservant des performances satisfaisantes de l'entraînement. Or, il se trouve que les machines polyphasées disposent de nombreuses phases ; on cherche donc à éviter de développer autant de modèles que de cas de dégradation possibles. En effet, une telle démarche conduirait à établir autant de structures de commande que de cas de dégradation qu'il faudrait organiser pour contrôler l'entraînement dans tous les cas de dysfonctionnement.

Pour éviter cette démarche jugée trop lourde, on cherche à établir un modèle unique de la machine auquel est adjoint un modèle de l'ouverture d'une phase d'alimentation qui soit totalement indépendant de la machine. Ces deux parties sont ensuite regroupées pour constituer un modèle unique de machine valable quelque soit son mode de fonctionnement. Une seule structure de commande peut alors être 
déduite offrant l'originalité de contrôler l'entraînement aussi bien en fonctionnement normal qu'en dégradé.

Pour atteindre cet objectif, la première partie de l'article met en place la modélisation vectorielle de l'entraînement électromécanique comportant une machine synchrone ou asynchrone triphasée en utilisant le formalisme vectoriel généralisé et la Représentation Energétique Macroscopique (REM). La modélisation de la dégradation de l'alimentation par ouverture d'une phase statorique utilise ce même formalisme pour conduire à un modèle complet de l'entraînement en mode dégradé. Une structure de commande en mode dégradé, fonctionnant aussi en mode normal, est déduite par inversion de la REM dans le cas de la machine synchrone, ou adaptée d'une commande industrielle existante dans le cas de la machine asynchrone. Chacun de ces cas est évalué au travers de simulations ; le cas de la machine asynchrone est validé expérimentalement. La dernière partie dresse un bilan des études et concerne l'adaptation de la méthode aux machines polyphasées alimentées par plus de trois phases.

\section{Modélisation de l'entraînement électromécanique}

\subsection{Hypothèses}

Les machines envisagées sont rotatives et triphasées à $p$ paires de pôles. Elles disposent de trois enroulements statoriques identiques de résistance électrique $R$ déphasées spatialement de $2 \pi / 3$. Chaque enroulement est alimenté par sa propre source de tension. Ce mode d'alimentation sans couplage électrique (figure 1) demeure peu répandu dans un contexte industriel, hormis dans les applications à haute sûreté de fonctionnement. Il est retenu ici pour contrôler le maximum de degrés de liberté (Ertugrul et al., 2002, Bennett et al., 2004 ; Baumann et al., 2007).

Pour se focaliser sur la dégradation de l'alimentation, le modèle de la machine est simplifié. Les matériaux magnétiques sont linéaires et sans pertes électromagnétiques. La géométrie des organes à l'origine du champ magnétique (encoches des bobinages statoriques et rotoriques, aimants, etc.) est sans effet sur l'entrefer dont l'épaisseur est constante. Enfin, on écarte les problèmes d'harmoniques occasionnés par :

- des convertisseurs à découpage (pleine onde, multiniveau ou à modulation de largeur d'impulsion);

- des f.m.m. non sinusoïdales.

En résumé, on s'attache à modéliser les phénomènes au premier harmonique. 


\subsection{Modélisation en mode normal}

\subsubsection{Alimentation de la machine}

Pour permettre l'exploitation de tous les degrés de liberté de la machine, les phases d'alimentation ne sont pas couplées électriquement. Chaque enroulement de la machine est alimenté par une source de tension monophasée. L'hypothèse de travail au premier harmonique permet de considérer les tensions et les courants purement sinusoïdaux. Les sources sont obtenues à partir des fonctions de modulation $m_{\mathrm{k}}$ avec $k \in\{a, b, c\}$ qui peuvent donc être modélisées par des amplificateurs linéaires continus alimentés par le bus continu $V_{\text {bus }}$ (Figure 1).

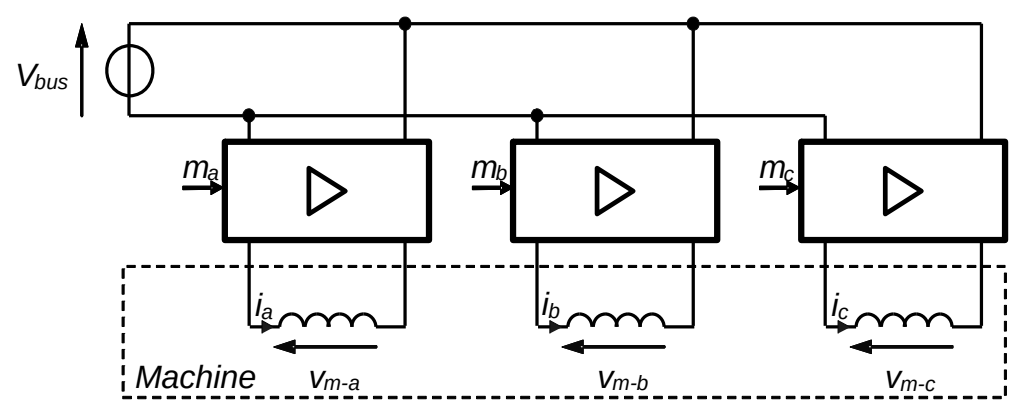

Figure 1. Alimentation de chaque phase par des sources indépendantes.

\subsubsection{L'actionneur : représentation vectorielle et machines fictives}

La modélisation de la machine utilise le formalisme vectoriel généralisé développé dans (Semail, 2000 ; Kestelyn, 2003). Il associe les grandeurs dans la machine à des vecteurs évoluant dans un espace vectoriel dont la dimension correspond au nombre de phases. Dans ce cadre, les grandeurs vectorielles liant les courants et les flux sont découplées en les exprimant dans des sous-espaces orthogonaux, ou sous-espaces propres (s.e.p.) de l'application linéaire associée.

Pour le cas des machines triphasées qui nous concernent, chacun des s.e.p. est de dimension 1 ou 2. Un espace de dimension 1 est associé à une machine électrique monophasée dite « homopolaire » $\mathrm{M}$ h, tandis que l'espace de dimension 2 est associé à une machine électrique diphasée dite «principale » $\mathrm{M}$ p. L'orthogonalité des s.e.p. correspond donc à une indépendance magnétique et électrique aux valeurs moyennes des machines fictives.

L'écriture des relations dans les sous-espaces propres établit la loi des mailles vectorielle au stator : 


$$
\vec{v}_{s}=R_{s} \cdot \vec{i}_{s}+\frac{\mathrm{d}}{\mathrm{d} t}\left(\vec{i}_{s}\right)+\vec{e}_{s r}[1]
$$

Où $\vec{e}_{s r}$ est le vecteur des forces électromotrices induites au stator par le rotor.

Une grandeur générique, représentée par un vecteur $\vec{x}$, se décompose dans les s.e.p. d'indice $h$ et $p$ par :

$$
\vec{x}=\overrightarrow{x_{h}}+\overrightarrow{x_{p}}[2]
$$

Le vecteur des tensions statoriques se décompose dans les machines fictives :

$$
\overrightarrow{v_{s}}=\overrightarrow{v_{h}}+\overrightarrow{v_{p}} \text { avec }\left\{\begin{array}{l}
\overrightarrow{v_{h}}=R_{s} \cdot \overrightarrow{i_{h}}+L_{h} \frac{\mathrm{d}}{\overrightarrow{i_{h}}}+\overrightarrow{e_{h}} \\
\overrightarrow{v_{p}}=R_{s} \cdot \overrightarrow{i_{p}}+L_{p} \frac{\mathrm{d}}{\mathrm{d} t} \overrightarrow{i_{p}}+\overrightarrow{e_{p}}
\end{array}\right.
$$

Le couple résultant total sur l'arbre de la machine s'écrit alors :

$$
C_{e m}=C_{h}+C_{p} \text { avec }\left\{\begin{array}{l}
C_{h}=\frac{1}{\Omega}\left(\overrightarrow{e_{h}} \cdot \overrightarrow{i_{h}}\right)=\frac{e_{h} \cdot i_{h}}{\Omega} \\
C_{p}=\frac{1}{\Omega}\left(\overrightarrow{e_{p}} \cdot \overrightarrow{i_{p}}\right)=\frac{e_{d} \cdot i_{d}+e_{q} \cdot i_{q}}{\Omega}
\end{array}\right.
$$

\subsubsection{Représentation Énergétique Macroscopique}

En suivant une représentation vectorielle, la mise en équation des machines a montré la coexistence de deux machines fictives magnétiquement indépendantes, mais couplées en amont à l'alimentation et en aval à la charge mécanique. Pour offrir une représentation unifiée des machines triphasées qui mette en évidence ces couplages, tout en offrant la possibilité ultérieure de déduire la commande de la machine par inversion, nous utilisons le formalisme de la Représentation Energétique Macroscopique (REM) présentée dans (Bouscayrol et al., 2000 ; Barre) et al., 2006) (figure 2). En partant de la gauche, on distingue la source électrique et le bloc de découplage des grandeurs dans les sous-espaces propres (changement de base obtenu par la transformation $\left.\quad T_{a b c-h d q}\right)$. A titre indicatif, cette transformation vectorielle peut utiliser les transformations matricielles de Concordia ou de Park (Semail et al ., 2004). Viennent ensuite deux chaînes parallèles comportant les machines fictives constituées du stockage d'énergie dans les enroulements (rectangle barré) suivi des convertisseurs électromécaniques $\mathrm{M}_{\mathrm{h}}$ et $\mathrm{M}_{\mathrm{p}}$. Les couples fictifs produits par chacune des machines sont ajoutés sur l'arbre réel. Vient enfin le stockage d'énergie dans l'arbre mécanique puis la charge mécanique à droite. 
Modélisation causale pour la commande auto adaptatée de machines en mode dégradé 9

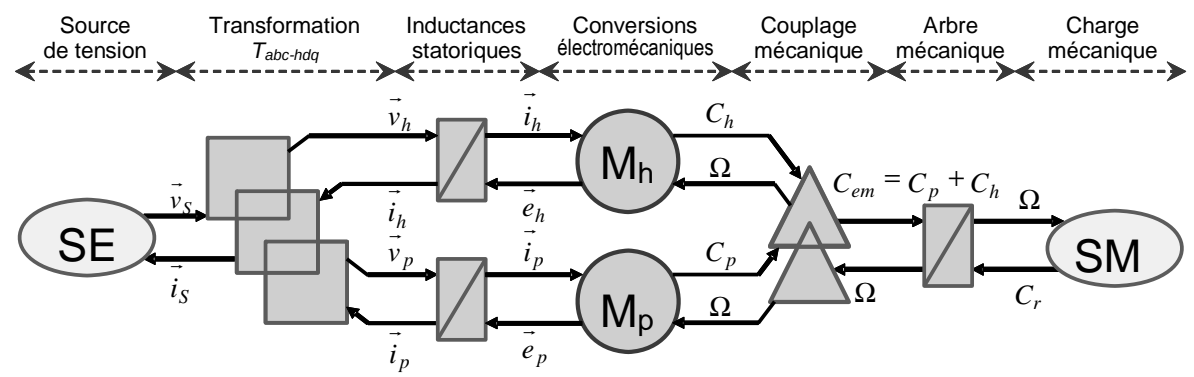

Figure 2. Modèle REM d'une machine triphasée.

Cette représentation synthétique regroupe les éléments principaux de la machine triphasée sans référence à sa technologie. Par contre, les expressions des f.e.m. dans les différentes machines fictives dépendent du champ rotorique et de l'interaction entre le stator et le rotor:

- le champ rotorique de la machine synchrone est produit indépendamment de l'alimentation du stator (flux rotorique constant);

- le champ rotorique de la machine asynchrone est induit par le champ statorique conjugué au mouvement du rotor. La mise en équation rappelée dans les annexes met en évidence cette interaction (Caron et al., 1995).

\subsubsection{Cas de la machine synchrone}

Dans le repère de Park, les vecteurs des f.e.m. statoriques et du flux statorique induit par le rotor s'écrivent :

$$
\vec{\phi}\left(\begin{array}{l}
\phi_{h} \\
\phi_{d} \\
\phi_{q}
\end{array}\right)_{I R} \text { et }\left.\frac{\mathrm{d} \phi}{\mathrm{d} t}\right|_{R}=\overrightarrow{e_{s r}}\left(\begin{array}{l}
e_{h} \\
e_{d} \\
e_{q}
\end{array}\right) \text { dans le repère de Park } R=\left(\underset{x_{h}, x_{d}, \overrightarrow{x_{q}}}{\longrightarrow}\right)_{[5]}
$$

On peut exprimer les composantes des f.e.m. statoriques sous la forme :

$$
\overrightarrow{e_{s r}}\left(\begin{array}{l}
e_{h}=p \cdot \Omega \cdot \phi_{h} \\
e_{d}=p \cdot \Omega \cdot \phi_{d} \\
e_{q}=p \cdot \Omega \cdot \phi_{q}
\end{array}\right)_{I R}
$$




\subsubsection{Cas de la machine asynchrone}

L'étude est limitée à la machine à rotor en court-circuit, ce qui exclut les cas de double alimentation. Les relations générales de fonctionnement sont d'abord classiquement écrites dans le repère de la base naturelle, puis transformées dans le repère de Park. Elles sont rappelées en annexe I. Ces relations mêlent des grandeurs relatives aux deux axes fictifs. Elles sont transformées afin de séparer l'écriture des grandeurs d'action (courants) et de réaction (f.e.m.) suivant chacun de ces axes $d$ et $q$ (annexe II). Enfin, en considérant que les tensions rotoriques sont nulles, la REM de la machine principale peut être tracée sur la figure 3 (Bouscayrol, 2003).

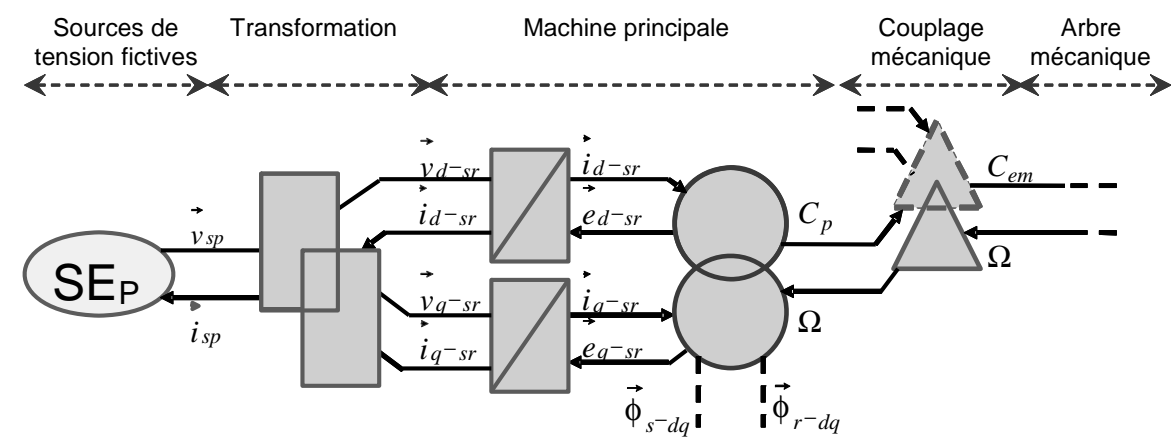

Figure 3. REM de la MAS limitée à la machine principale.

Les flux magnétiques jouent un rôle notable dans l'élaboration des f.e.m. Pour les percevoir plus aisément sur la représentation, ces grandeurs sont indiquées par des traits interrompus au niveau du couplage magnétique.

\subsection{Modélisation en mode dégradé}

La situation provoquant le mode dégradé retenu dans ce document peut provenir d'un défaut d'ouverture et/ou de fermeture d'un interrupteur de l'onduleur ou d'un défaut dans la liaison machine-onduleur (défaut de connexion, déclenchement d'une protection, etc.). Elle conduit à l'ouverture d'une phase d'alimentation statorique caractérisée par l'annulation du courant. La modélisation s'articule autour de deux approches.

D'abord, un nouveau modèle en mode dégradé peut être établi (Zhao, 1996a et 1996b ; Ryu et al. 2004a et 2004b). Une machine en mode dégradé est alors traitée comme une machine déséquilibrée avec une ou plusieurs phases en moins. Cette solution établit autant de modèles de la machine que de dégradations possibles. Le comportement global est perçu au travers de la commutation des différents modèles. 
Cependant, puisque l'on souhaite établir une méthode générale applicable à toutes les machines polyphasées, cette solution n'est pas retenue dans notre cas en raison du nombre important de configurations résultant de la combinaison du nombre de phases et des cas de dégradation.

L'autre solution consiste alors à ne pas changer le modèle de la machine, mais à $\mathrm{y}$ adjoindre le modèle de la phase ouverte.

\subsubsection{Recherche d'un modèle de la dégradation}

L'ouverture d'une phase peut être décrite par l'insertion :

- d'un interrupteur parfait pour représenter la déconnexion de la phase ;

ou

- d'une résistance électrique dont la valeur caractérise l'état de la phase.

Avec la première solution, l'utilisation d'un interrupteur conduit à envisager la situation illustrée sur la figure 4.

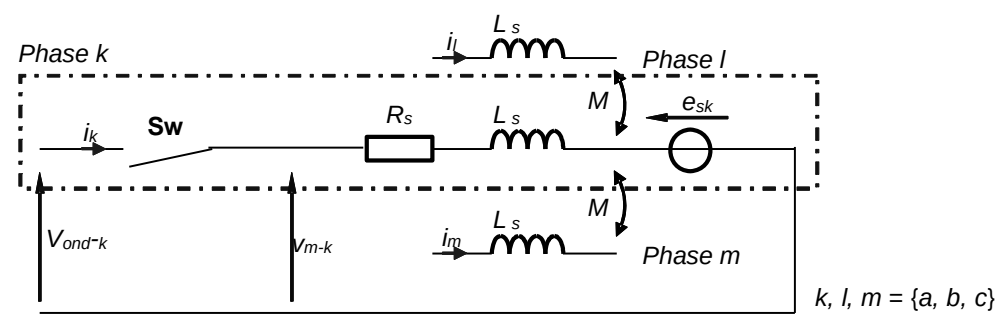

Figure 4. Phase ouverte par la dégradation.

Dans le cas général, la tension machine de la phase $k$ dépend des courants dans les phases $l$ et $m$ :

$$
v_{m^{-} k}=R_{s} \cdot i_{k}+L_{s} \frac{\mathrm{d} i_{k}}{\mathrm{~d} t}+M \frac{\mathrm{d} i_{l}}{\mathrm{~d} t}+M \frac{\mathrm{d} i_{m}}{\mathrm{~d} t}+e_{s k}[7]
$$

Si la phase $k$ est ouverte, $i_{\mathrm{k}}=0$; on observe alors la tension $v_{\mathrm{m}-\mathrm{k}}$ aux bornes de l'enroulement de la machine :

$$
v_{m-k}=e_{s k}+M \frac{\mathrm{d} i_{l}}{\mathrm{~d} t}+M \frac{\mathrm{d} i_{m}}{\mathrm{~d} t}[8]
$$


Avec cette relation, dès qu'une phase s'ouvre, la tension $v_{\mathrm{m}-\mathrm{k}}$ n'est plus imposée par la source mais par l'enroulement de la machine en défaut. Un régime de « générateur non contrôlé » (UnControlled Generator ou UCG) s'installe (Welchko et al., 2002). Le choix d'une modélisation en causalité intégrale qui favoriserait la déduction de la commande ne peut être respecté ici puisque la tension $v_{\mathrm{m}-\mathrm{k}} \mathrm{s}$ 'exprime à partir de la dérivée des courants dans les phases saines. C'est pour cette raison que la représentation de la dégradation par l'ouverture d'un interrupteur est écartée.

Ce problème nous a conduits à opter pour la seconde approche. L'élément inséré, que nous appelons « dipôle ouvrant » (DO), est en fait une résistance (figure 5) de valeur nulle lorsque la phase est saine, mais devenant très grande en cas d'ouverture ${ }^{1}$ (Kestelyn et al., 2007). L'ouverture de la phase se réduit alors à la variation paramétrique de la valeur de la résistance.

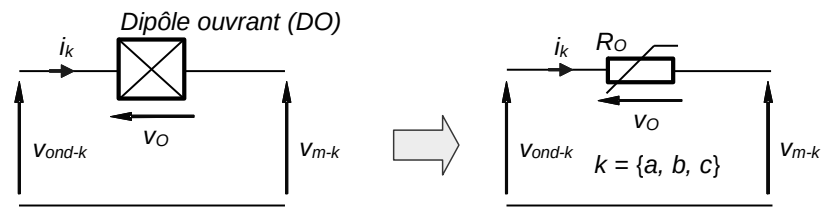

Figure 5. Dipôle ouvrant matérialisant l'ouverture d'une phase statorique.

Cette méthode avait déjà été utilisée sous l'appellation de « resistive companion method» (RCM) dans le solveur Virtual Test Bed (VTB) décrit dans (Monti, 2001).

Pour caractériser la REM de cet élément, on exprime les tensions sur les mailles :

$$
\left[\begin{array}{l}
v_{m^{-} a} \\
v_{m-b} \\
v_{m^{-} c}
\end{array}\right]=\left[\begin{array}{l}
v_{\text {ond }}-a \\
v_{\text {ond }}-b \\
v_{\text {ond }}-c
\end{array}\right]-\left[\begin{array}{ccc}
R_{O} & 0 & 0 \\
0 & R_{O} & 0 \\
0 & 0 & R_{O}
\end{array}\right] \cdot\left[\begin{array}{l}
i_{a} \\
i_{b} \\
i_{c}
\end{array}\right] \text { [9] }
$$

Cette relation traduit que la tension appliquée à une phase est dépendante de celle aux bornes de l'élément ouvrant DO, ce qui se traduit par un couplage représenté sur la REM de la figure 6.

Le modèle de l'ouverture obtenu pour une seule phase est valable aussi bien en fonctionnement normal, qu'en dégradé. Il est de plus indépendant de celui de la machine. Il est alors possible de l'associer à ce dernier.

\footnotetext{
${ }^{1}$ La résistance est idéalement infinie, mais prend une valeur finie pour les simulations.
} 


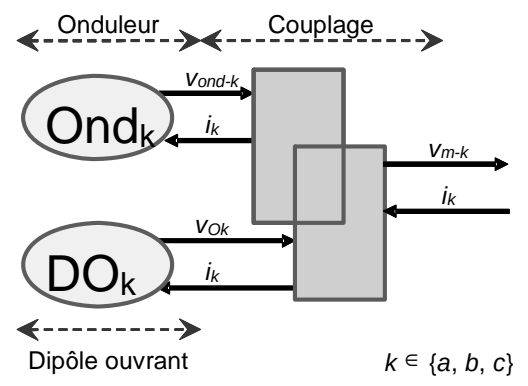

Figure 6. REM d'une dégradation par un élément ouvrant DO dans la phase $k$.

\subsubsection{Insertion de la dégradation dans l'entraînement}

Avec l'introduction des dipôles ouvrants sur chaque phase, le modèle est étendu à l'ensemble de la machine. En se limitant à l'alimentation, le modèle global utilisant la REM est représenté sur la figure 7. Le couplage mécanique et l'arbre de la machine ne sont pas modifiés par rapport à la figure 2 et n'apparaissent plus sur la nouvelle figure.

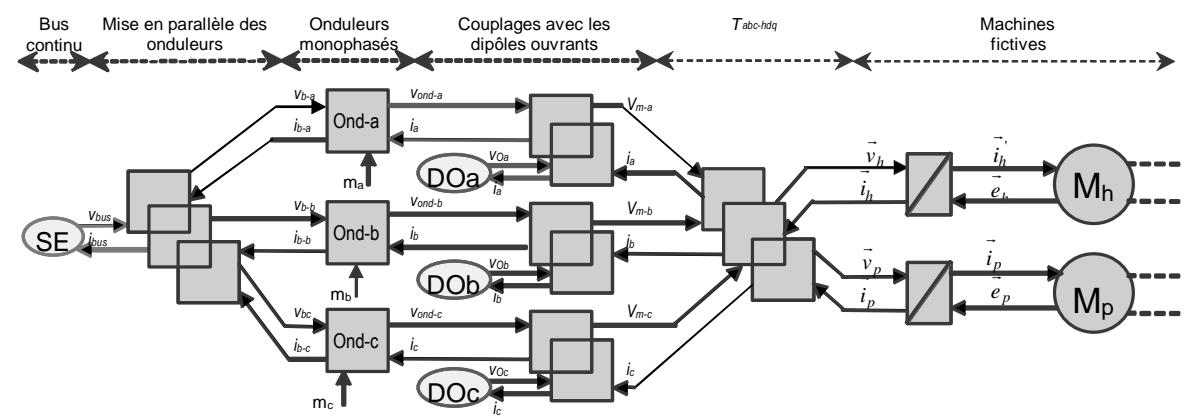

Figure 7. REM de l'entraînement sans la partie mécanique.

L'ensemble ainsi mis en place constitue un modèle unique valable pour décrire le fonctionnement en mode normal aussi bien que dégradé.

2.3.3. Effets d'une dégradation sur les grandeurs des machines fictives

2.3.3.1. Transformation du modèle

Bien qu'achevé, le modèle de l'entraînement ne permet pas encore d'apprécier l'influence d'un dipôle ouvrant sur les grandeurs dans les machines fictives. Grâce à 
la linéarité de la transformation $T_{a b c-h d q}$, on peut effectuer la permutation de ce bloc avec ceux des $\mathrm{DO}_{\mathrm{k}}$. Le résultat conduit à la nouvelle REM sur la figure 8 .

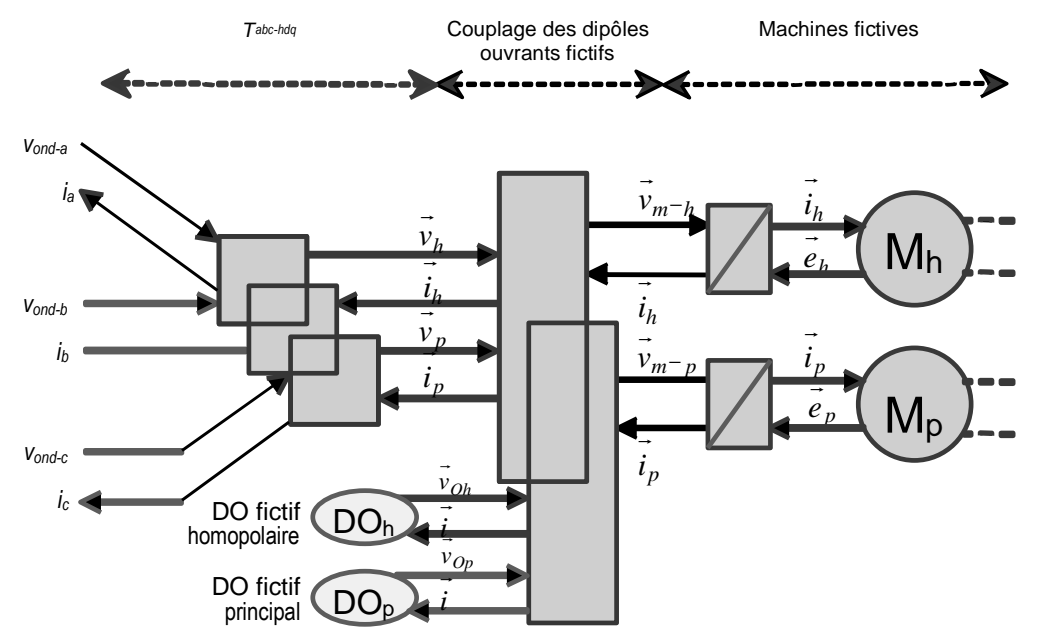

Figure 8. REM partielle de l'entraînement limitée aux couplages électriques.

Avec cette modification introduite graphiquement par la REM, les relations peuvent maintenant être directement exprimées dans les machines fictives.

\subsubsection{Mise en équation des tensions et conséquences sur les courants}

La relation [9] qui traduit le couplage est réécrite en utilisant la transformation de Park $T_{a b c-h d q},(\mathrm{Cf}$. annexe IV), ce qui se traduit par :

$$
\left[\begin{array}{c}
v_{m^{-} h} \\
v_{m^{-} d} \\
v_{m^{-} q}
\end{array}\right]=\left[\begin{array}{c}
v_{\text {ond }^{-} h} \\
v_{\text {ond }^{-} d} \\
v_{\text {ond }^{-} q}
\end{array}\right]-\left[\begin{array}{ccc}
R_{O h} & R_{\text {Ohd }} & R_{\text {Ohq }} \\
R_{\text {Ohd }} & R_{\text {Od }} & R_{\text {Odq }} \\
R_{\text {Ohq }} & R_{\text {Odq }} & R_{O q}
\end{array}\right] \cdot\left[\begin{array}{c}
i_{h} \\
i_{d} \\
i_{q}
\end{array}\right][10]
$$

L'analyse de cette relation montre que cette fois, ce sont les tensions aux bornes des machines fictives qui dépendent directement de l'état de DO k. La matrice de la dégradation représentant les couplages de chaque phase avec les dipôles ouvrants est diagonale dans la base naturelle. Elle se transforme en une matrice pleine, dite fictive dans les sous-espaces propres. Les coefficients $R_{\text {Oxy }}$ décrits en annexe IV se regroupent en trois catégories : 
$-R_{\text {Oh }}$ est une résistance fictive constante ;

- $R_{\text {Ohd }}$ et $R_{\text {Ohq }}$ sont des fonctions sinusoïdales de l'angle électrique $\quad p^{\theta}$ (où $\theta$ repère classiquement la position rotor : $\theta=\omega_{t / p}$ );

- $R_{\mathrm{Od}}, R_{\mathrm{Oq}}$ et $R_{\mathrm{Odq}}$ sont des fonctions sinusoïdales de l'angle électrique $2 p^{\theta}$.

L'écriture de la matrice montre que les machines fictives, indépendantes en mode normal, sont couplées en fonctionnement dégradé. La dépendance des coefficients avec l'angle électrique indique que des harmoniques de tension vont apparaitre et perturber les machines fictives en induisant des courants variables.

Plus précisément, la relation [10] et l'expression des coefficients de la matrice fictive montrent que si des courants constants sont maintenus dans les machines fictives, le couple pourra être constant, mais il faut alors que les tensions délivrées par les onduleurs fictifs ( $v_{\text {ond-x }}$ ) soient variables. A contrario, si les tensions issues de l'onduleur sont constantes, les courants sont variables avec l'angle $p^{\theta}$ (fondamental à $f$ ) dans $\mathbf{M}_{\mathrm{h}}$ et $2 p^{\theta}($ à $2 f)$ dans $\mathbf{M}_{\mathrm{p}}$; ils seront alors responsables des ondulations de couple à cette même fréquence.

\subsection{Conclusion sur la modélisation}

Lors d'une dégradation de l'alimentation d'une machine alternative par l'ouverture d'une phase statorique, la machine n'est pas modifiée. Puisqu'elle reste saine, son modèle ne change pas. Il faut alors modéliser l'ouverture de la phase en défaut avec un bloc supplémentaire assurant le couplage entre la source et la machine. Les deux modèles obtenus sont associés pour obtenir une description unique, valable à la fois quand la machine est alimentée correctement, mais aussi quand un défaut ouvre l'une des phases.

Ce nouveau modèle montre que la dégradation introduit une matrice de résistances fictives variables. L'examen des termes de cette matrice de couplage met en évidence qu'il peut exister des ondulations des courants fictifs dans la machine principale à deux fois la fréquence fondamentale $f$ des grandeurs statoriques. En combinant ces courants ondulés à des f.e.m. constantes dans les machines fictives (machines à f.e.m. sinusoïdales), des pulsations de couple apparaissent à la même fréquence, c'est-à-dire $2 f$.

Pour envisager la correction de couple, il faut éliminer les harmoniques de courant qui apparaissent dans les machines fictives. C'est l'objet du paragraphe suivant. 


\section{Correction du couple en cas d'ouverture d'une phase statorique}

\subsection{Stratégie de contrôle des courants}

En cas d'ouverture d'une phase statorique de machine synchrone ou asynchrone à f.e.m. sinusoïdales, les ondulations de couple s'expliquent par le fait que les courants fictifs ne sont plus constants en régime permanent. Rappelons aussi, avec les hypothèses choisies, que la f.e.m. est constante dans la machine principale et nulle dans la machine homopolaire. Cette dernière ne produit donc aucun couple quelque soit le courant l'alimentant. Cette analyse du comportement des machines fictives permet de préciser où doit porter la correction. Avec le bilan des harmoniques de courant en fonctionnement dégradé, il devient possible d'envisager le mode d'action sur les grandeurs fictives pour éliminer l'effet de la perturbation. Restera alors à établir le moyen le plus approprié pour atteindre cet objectif puis d'en apprécier l'efficacité.

Avec nos hypothèses de travail, le stator de la machine triphasée en fonctionnement normal est alimenté par trois tensions indépendantes et en retour la machine produit les trois courants statoriques. L'entraînement dispose de trois degrés de liberté qui se manifestent au travers de trois grandeurs indépendantes : deux pour produire le champ tournant et la troisième non fixée a priori (action sur les pertes Joule par exemple). L'ouverture d'une phase due à un défaut annule un courant statorique : la commande ne peut plus agir sur cette grandeur et le système perd un degré de liberté par rapport aux trois initialement disponibles. En gérant ces deux degrés de liberté, la commande doit abandonner le contrôle d'une grandeur qui, par conséquent, n'est plus asservie.

Dans la base naturelle, l'annulation du coụrant dans la phase dégradée fait perdre une composante au vecteur courant $\vec{i}_{s}$. Par exemple, quand $i_{\mathrm{a}}$ s'annule, $\vec{i}_{s}$ s'exprime par:

$$
\overrightarrow{i_{s}}=0 \cdot \vec{x}_{a}+i_{b} \cdot \vec{x}_{b}+i_{c} \cdot \vec{x}_{c} \text { dans la base naturelle }
$$

Le courant s'écrit donc aussi :

$$
\vec{i}_{s}=i_{h} \cdot \vec{x}_{h}+i_{d} \cdot \vec{x}_{d}+i_{q} \cdot \vec{x}_{q} \text { dans la base des machines fictives }
$$

Mais deux composantes parmi $\quad i_{\mathrm{h}}, i_{\mathrm{d}}$ et $i_{\mathrm{q}}$ sont suffisantes pour exprimer la troisième (c'est une combinaison linéaire des deux autres). La figure 9 illustre la dépendance des composantes restantes quand le courant dans la phase $a$ s'annule et que l'on désire conserver le même vecteur courant dans la machine principale. Les composantes du vecteur courant dégradé sont obtenues : 
- par projection du vecteur dans le plan d'équation $i_{\mathrm{a}}=0$, ce qui correspond aux deux composantes réelles $i_{\mathrm{b}}$ et $i_{\mathrm{c}}$;

- par projection du vecteur sur l'axe homopolaire (1 degré de liberté) et dans le plan de la machine principale avec, dans ce cas, la contrainte de rester sur un cercle, correspondant à un degré de liberté qui est l'angle de rotation autour de l'axe homopolaire.

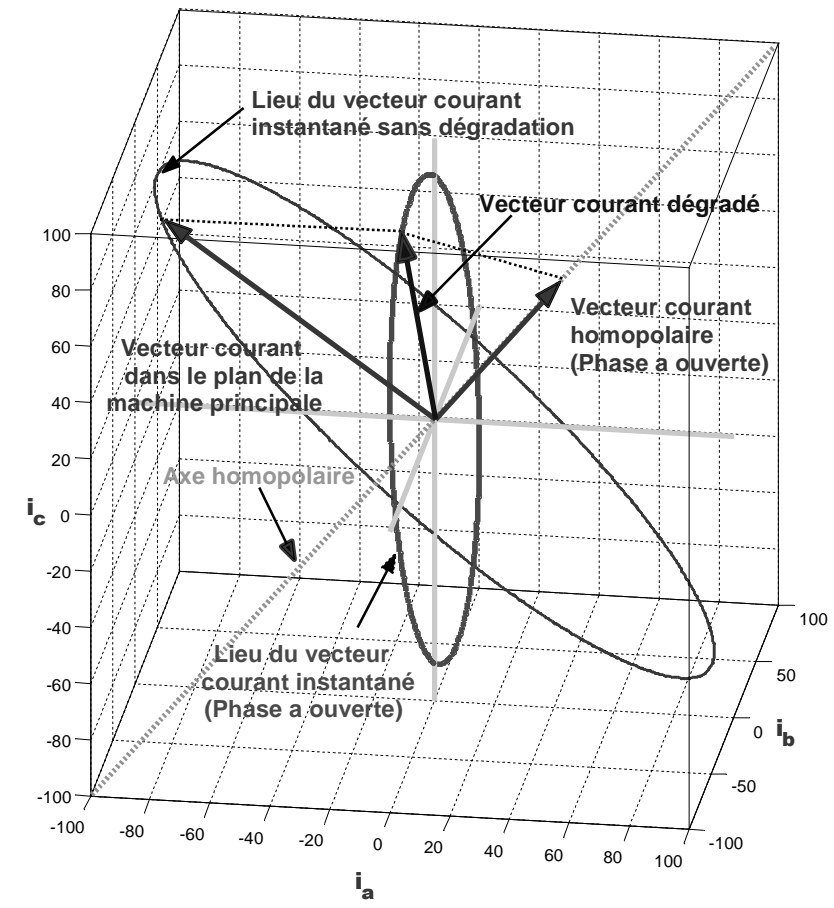

Figure 9. Représentation du vecteur courant dégradé dans deux repères différents.

Cette représentation montre que le vecteur courant dégradé nécessite deux composantes indépendantes, même si elles sont exprimées dans des repères différents. La perte d'un degré de liberté peut aussi être mise en évidence par l'évaluation du courant homopolaire quand un courant réel s'annule dans une phase. Ce courant est obtenu en appliquant la transformation $\quad T_{a b c-h d q}$ au vecteur des courants réels lorsque l'un d'eux ( $i_{\mathrm{a}}, i_{\mathrm{b}}$ ou $i_{\mathrm{c}}$ ) est nul. On en déduit alors son expression :

$$
i_{s h}=\sqrt{2}\left[\mathbf{i}_{s q} \sin \left(p^{\theta}-\alpha\right)-i_{s d} \cos \left(p^{\theta}-\alpha\right)\right]_{[13]}
$$


L'angle $\alpha$ dépend de la phase $k$ ouverte $(k \in\{1$ (a), 2 (b), 3 (c) $\}): \quad \alpha=2 \pi_{(k-}$ $1) / 3$.

Ce constat de dépendance des grandeurs permet d'en déduire la grandeur qui ne sera plus asservie. La répartition en machines fictives de dimension 1 ou 2 fournit une aide à la réponse :

- ne plus asservir le courant de la machine homopolaire en libérant le seul degré de liberté de cette machine. Le courant devenu non nul ne produira aucun couple puisque la f.e.m. de cette machine est nulle ;

- abandonner le contrôle d'une seule composante du courant dans M p. Suivant les machines, cette solution n'est pas toujours envisageable. Pour une machine synchrone, seul $i_{\text {d }}$ peut être libéré puisque le couple ne dépend que de $i_{\mathrm{q}}$. Mais la perte de contrôle de ce courant peut entraîner la démagnétisation des aimants. Cette solution n'est pas envisageable pour une machine asynchrone qui nécessite d'être magnétisée.

Pour que la solution soit générale, nous choisissons de ne plus asservir le courant homopolaire en cas de dégradation.

Pour apprécier la validité de la méthode appliquée aux principales machines triphasées, la stratégie de contrôle est d'abord mise en place sur la machine synchrone. Ce choix a pour but de mettre en œuvre la méthode avec une machine plus simple à modéliser et à commander. Les résultats obtenus sont ensuite adaptés à la machine asynchrone qui requiert une structure de commande plus élaborée.

\subsection{Cas de la machine synchrone}

\subsubsection{Mise en place d'une commande en mode dégradé}

La structure maximale de commande (SMC) de la machine est obtenue par l'inversion systématique de la REM pour fournir un modèle de commande générique indépendant du mode de fonctionnement retenu. Le résultat donné sur la figure 10 est valable en mode normal comme en mode dégradé. 


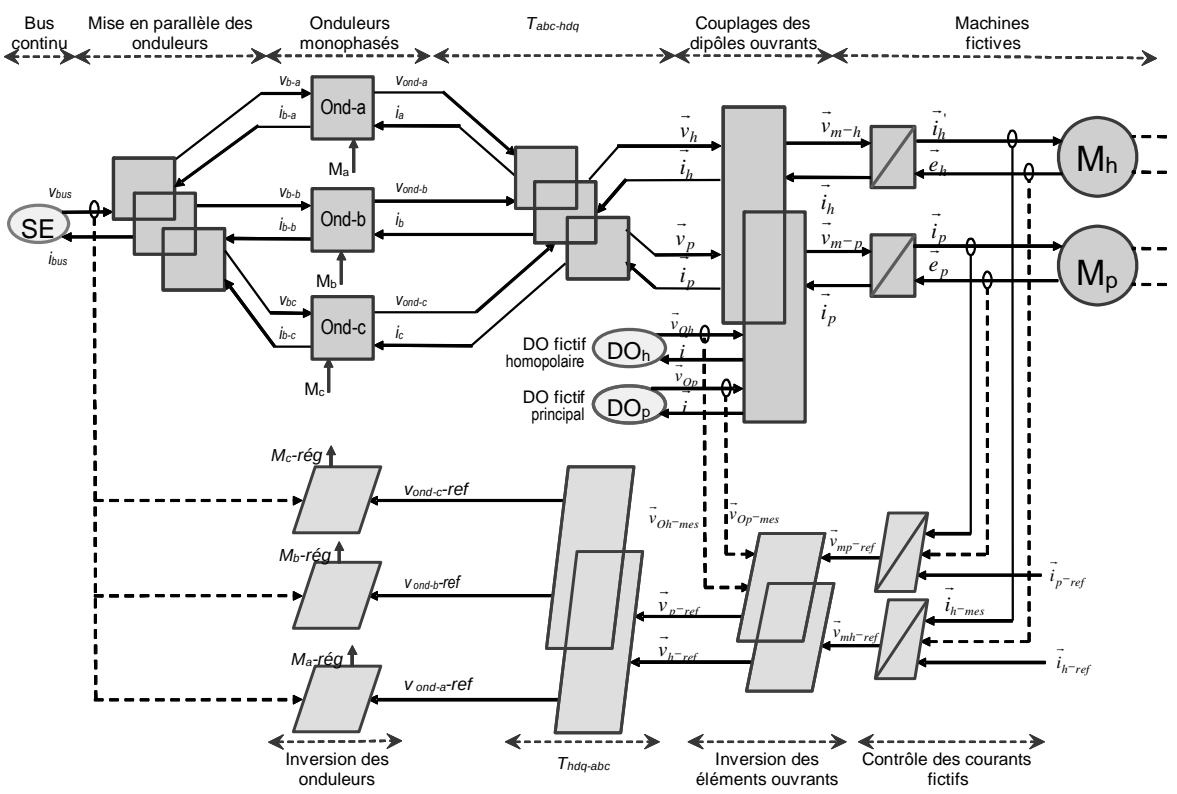

Figure 10. REM et SMC limitées aux machines fictives.

Aux blocs décrivant la REM, à lire de la gauche vers la droite, sont adjoints des blocs en miroir décrivant la commande, à lire de la droite vers la gauche :

- un parallélogramme barré correspond à une inversion indirecte mettant en œuvre un asservissement de la grandeur associée ;

- une association de parallélogrammes imbriqués élabore le découplage des grandeurs liées dans le bloc de couplage associé ;

- un parallélogramme élabore la consigne du convertisseur (carré) associé ;

Les grandeurs mesurées sur le système (capteurs) sont repérées par des ellipses, tandis que l'information transmise est tracée par une ligne :

- en trait plein pour les grandeurs à prendre obligatoirement en compte ;

- en traits pointillés pour les grandeurs dont la prise en compte est facultative.

La structure de commande issue de la REM référence tous les moyens d'action possibles sur les machines fictives. Si on se limite à $\mathrm{M}_{\mathrm{p}}$ conformément à la stratégie adoptée, l'action peut se porter sur l'asservissement des deux composantes $i_{\mathrm{d}}$ et $i_{\mathrm{q}} \mathrm{du}$ courant (trait plein à gauche de $\mathrm{M}_{\mathrm{p}}$ ). En fonction de la performance des correcteurs et de leur capacité à rejeter les perturbations, on peut éventuellement compenser les f.e.m. (traits interrompus). La compensation des tensions $V_{\text {Oh }}$ et $V_{\text {Op }}$ aux bornes des éléments ouvrants fictifs $\left(\mathrm{DO}_{\mathrm{h}}\right.$ et $\mathrm{DO}_{\mathrm{p}}$ ) peut également être effectuée en retranchant 
la tension fictive qui apparaît aux bornes de l'élément ouvrant lorsque la phase s'ouvre. Pour la connaître, un capteur de tension doit être en mesure de prélever la tension réelle aux bornes de la liaison électrique entre chaque bras d'onduleur et la borne de l'enroulement correspondant. Cette méthode a été étudiée uniquement en simulation sans être approfondie par des vérifications pratiques, principalement pour éviter la mise au point des chaînes de mesure de tension spécifiques. Elle n'est pas développée dans ce document.

Sur cette base, la suite de l'étude repose sur l'asservissement des courants dans les machines fictives.

\subsubsection{Analyse de l'effet d'un défaut sur des correcteurs classiques}

Les correcteurs implantés dans la commande (figure 11) pour contrôler les 3 courants $i_{\mathrm{h}}, i_{\mathrm{d}}$ et $i_{\mathrm{q}}$ sont classiquement de type proportionnel et intégral (PI).

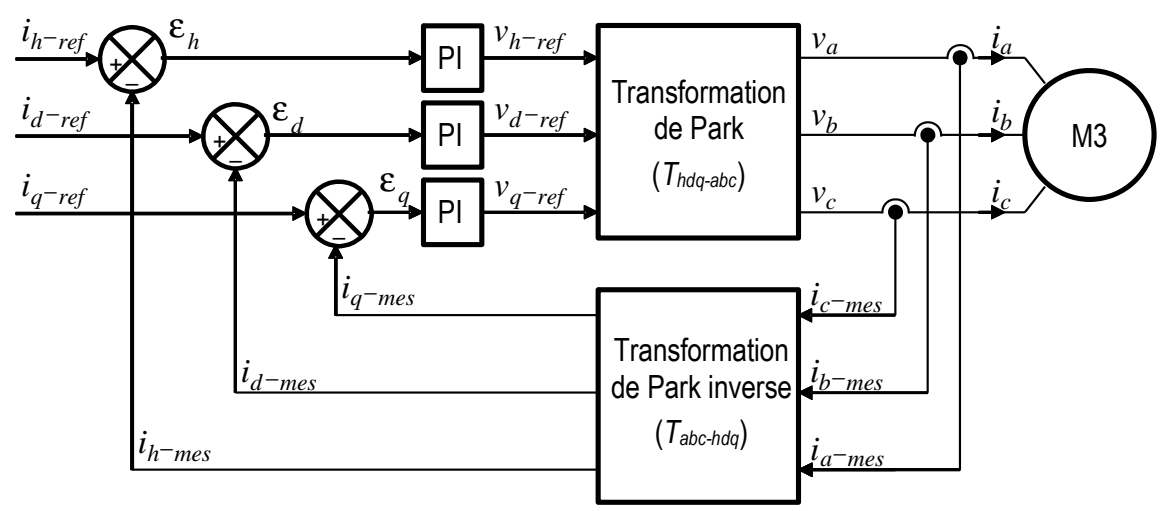

Figure 11. Les correcteurs PI dans les asservissements de courant.

Il est nécessaire d'analyser le mode d'action des correcteurs PI en cas de dégradation. Pour cela on considère dans un premier temps des correcteurs parfaits : un gain très élevé (infini) pour la fréquence nulle et un gain nul pour les fréquences strictement positives (figure 12a). Ils ne préservent donc que les composantes continues.

Avec une alimentation saine, les courants fictifs estimés $\quad i_{\mathrm{x} \text {-mes }}$ (où $x=h, d$ ou $q$ ) en régime permanent sont constants et les erreurs statiques $\varepsilon_{\mathrm{x}}$ sont nulles puisque les PI ont un gain statique infini. Par conséquent, les courants dans les phases sont sinusoïdaux et le couple est constant. 

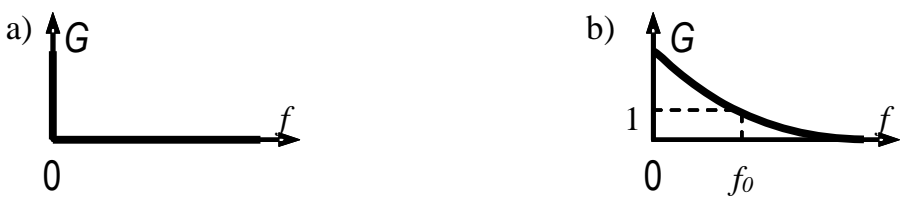

Figure 12. Réponses fréquentielles d'un correcteur PI : a) parfait et b) réel .

Quand un courant s'annule dans une phase, l'analyse de la relation [10] a montré que les courants fictifs dans les machines fictives sont variables. Si les consignes $i_{\mathrm{x}-}$ ref restent constantes, les erreurs $\varepsilon_{\mathrm{x}}$ comportent alors les mêmes harmoniques que les courants ; les correcteurs les rejettent parfaitement. Les consignes de tension fictives $v_{\mathrm{x} \text {-ref }}$ constantes sont préservées. Ceci induit des tensions onduleur $v_{\mathrm{y}}$ (où $y=a, b$ ou c) sinusoïdales, mais non équilibrées aux bornes de la machine en raison de l'ouverture de l'une des phases. À terme, les courants sont sinusoïdaux dans les deux phases saines et par conséquent le couple est ondulé.

Pour les correcteurs réels, la figure $12 \mathrm{~b}$ montre la réponse approchée du gain. Sa décroissance progressive atténue les fréquences au-delà de sa fréquence de transition $f_{0}$ sans toutefois les annuler. Les harmoniques à la fréquence $2 f$ résultant de la dégradation sont d'autant mieux rejetés que $f$ est faible. Mais en demeurant, ces harmoniques ne permettent qu'un lissage imparfait du couple. Ainsi, lorsque la vitesse de rotation est faible, les harmoniques se situent dans la bande passante du correcteur, si bien que le couple est peu ondulé. Lorsque la vitesse est élevée, les harmoniques sont dans une bande de fréquence dans laquelle le correcteur n'agit pratiquement pas ; les perturbations sont mal rejetées et le couple reste ondulé. Si l'impact sur la vitesse peut alors, de ce fait, être acceptable, les ondulations de couples sont elles bien présentes, sollicitant mécaniquement les accouplements de l'entraînement.

En résumé, le correcteur PI ne réduit pas de manière satisfaisante les ondulations de couple en régime dégradé d'alimentation sur une large plage de fréquences. Pour obtenir de meilleurs résultats et puisque la fréquence des perturbations est connue, il faut un correcteur capable de rejeter cet harmonique (ici à $2 f$ ), c'est-à-dire disposant également d'une amplification infinie à cette fréquence.

\subsubsection{Amélioration de l'action du correcteur PI}

La solution retenue pour éliminer efficacement les ondulations à la fréquence $2 f$ consiste à utiliser en parallèle sur la structure PI, un correcteur résonant dont la réponse en fréquence est donnée sur la figure 13 (Wulveryck, 2000). 


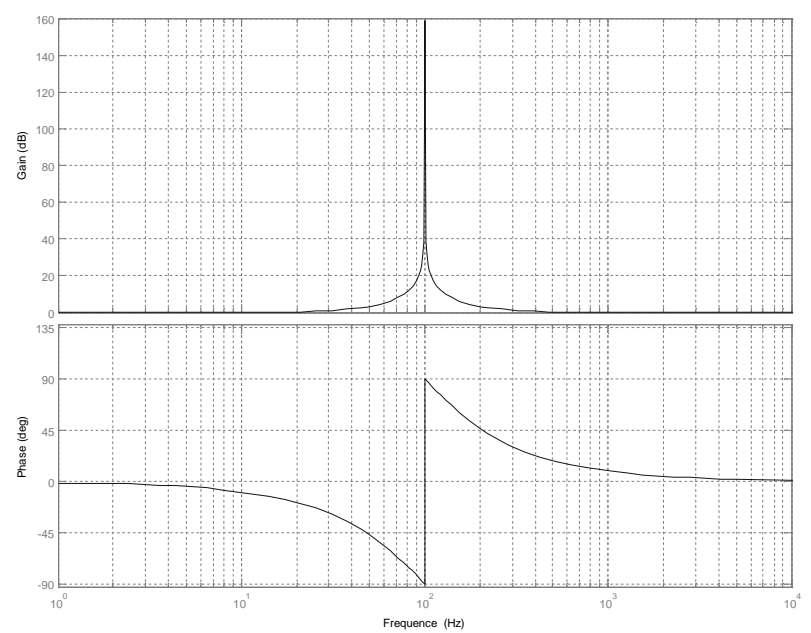

Figure 13. Diagrammes de Bode d'un correcteur résonant (ici à $100 \mathrm{~Hz}$ ).

La nouvelle structure de correction est illustrée figure 14. Chacune des voies est traitée par un correcteur global résultant de l'association d'un correcteur résonant placé en parallèle d'un correcteur PI. Les fonctions de transfert y sont aussi indiquées ( $s$ est la variable de Laplace).

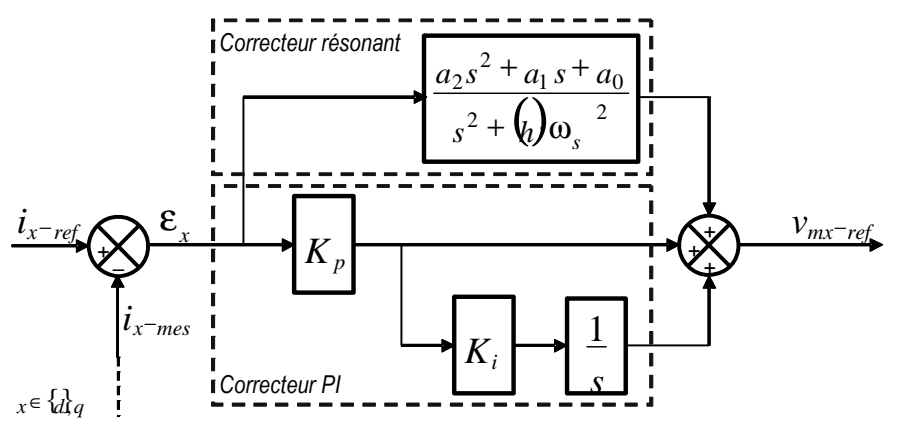

Figure 14. Usage d'un correcteur résonant en parallèle sur le PI.

Afin de disposer d'une structure de correction naturellement adaptée, c'est à dire qui fonctionne aussi bien en régime normal que dégradé, nous devons maintenant régler les correcteurs PI et résonant de façon à ce que le correcteur PI agisse en 
fonctionnement normal, tandis que le résonant n'agisse qu'en cas de dégradation par insertion d'un gain infini à la fréquence de la perturbation considérée.

\subsubsection{Réglage global du correcteur PI+résonant}

Pour contrôler les deux courants $i_{\mathrm{d}}$ et $i_{\mathrm{q}}$ de la machine principale, la commande nécessite d'utiliser deux correcteurs. À partir de la figure 14, chacun de ces correcteurs dispose d'une fonction de transfert de la forme :

$$
C(s)=C_{P I}(s)+C_{R}(s)=K_{p} \frac{1+\tau_{i} s}{\tau_{i} s}+\frac{b_{2} s^{2}+b_{1} s+b_{0}}{s^{2}+\omega_{p}^{2}}[14]
$$

Le réglage doit prendre en compte la fonction de transfert globale :

$$
C(s)=\frac{d_{3} s^{3}+d_{2} s^{2}+d_{1} s+d_{0}}{c_{3} s^{3}+c_{2} s^{2}+c_{1} s+c_{0}}
$$

Le paramètre de réglage du correcteur résonant est sa fréquence d'accord $f$ o qui dépend de la vitesse de rotation de l'arbre. Par conséquent, l'expression des coefficients $c_{0}, c_{1}, c_{2}, c_{3}$ et $d_{0}, d_{1}, d_{2}, d_{3}$ s'exprime avec $f_{0}$, ce qui nécessite que la commande les évalue en temps réel.

De bonnes performances du système peuvent, par exemple, être obtenues avec un réglage par placement des pôles utilisant le critère de la marge de stabilité généralisée ou MSG (Zheng et al., 2004).

\subsubsection{Vérifications en simulation}

L'implantation du modèle d'une machine (2 paires de pôles, $1500 \mathrm{tr} / \mathrm{min}$, tension nominale de $100 \mathrm{~V}$ et puissance nominale de $750 \mathrm{~W}$ ) et de sa commande a été réalisée sous MatLab Simulink ${ }^{\mathrm{TM}}$.

Les simulations sont faites à la vitesse constante de $1500 \mathrm{tr} / \mathrm{min}$. Quand une phase est ouverte, la figure 15 montre qu'une ondulation résiduelle importante demeure en raison d'une action PI insuffisante. Si une action PI+résonante est mise en place, les ondulations de couple se réduisent et tendent à s'annuler (partie droite de la figure 15). 


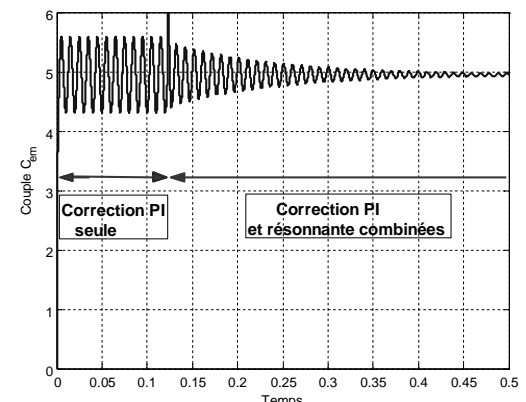

Figure 15. Effet de la correction sur le couple produit par la machine.

L'apparition du courant homopolaire $i$ h en cas de dégradation est visible sur la figure 16 gauche. La figure 16 droite montre la réduction des ondulations des courants dans la machine principale qui conduit à l'évolution vers un couple constant.
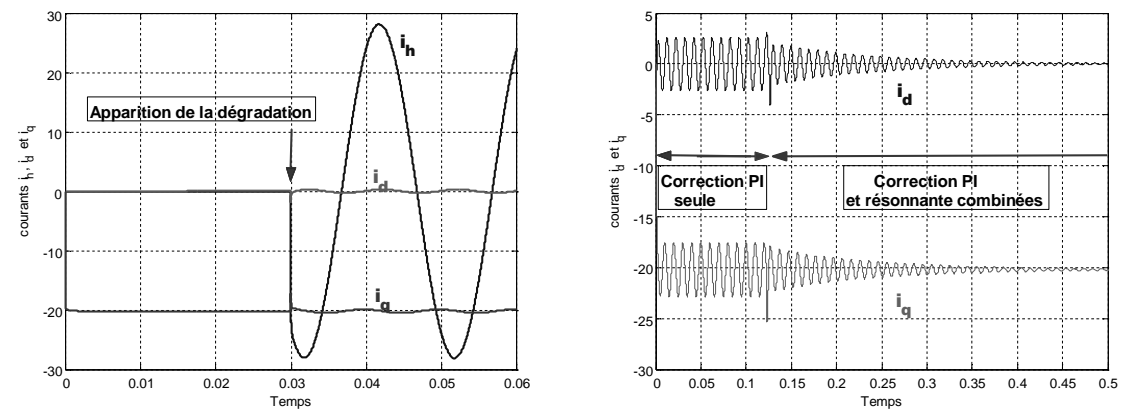

Figure 16. Action des correcteurs PI+résonant.

\subsubsection{Bilan et conclusion}

L'application de la méthode de réduction des pulsations de couple à la machine synchrone donne des résultats satisfaisants quelque soit la phase en défaut. En effet, la phase ouverte ne modifie que le déphasage des courants dans les machines fictives. Comme le correcteur utilisé n'y est pas sensible, le résultat obtenu demeure indépendant du numéro de phase ouverte.

La méthode peut maintenant être étendue à la machine asynchrone pour montrer son caractère général. 


\subsection{Cas de la machine asynchrone}

Avec cette machine, il est nécessaire de distinguer les grandeurs au stator, affectées de l'indice $« s »$, de celles au rotor, affectées de l'indice « $r »$.

3.3.1. Mise en place d'une commande en mode dégradé

Parmi toutes les commandes possibles visant à contrôler le couple (Boldea et al., 1992 ; Caron et al., 1995 ; De Fornel, 2004), la commande vectorielle indirecte à flux rotorique orienté (IRFO) est retenue ici pour sa simplicité de mise en œuvre.

Avec cette commande, le couple est donné par :

$$
T_{p}={ }_{p} \frac{M}{L_{r}} \phi_{r d} \cdot i_{s q}=\frac{1}{F_{q}} \phi_{r d} \cdot i_{s q} \text { (M l'inductance mutuelle stator-rotor) }
$$

Le flux $\phi_{\text {rd }}$ s'exprime par :

$$
\phi_{r d}=\frac{M}{1+\tau_{r} s} i_{s d} \text { avec } \tau_{r}=L ! \quad R_{r} \text { la constante de temps rotorique }
$$

En maintenant $\phi_{\text {rd }}$ constant par l'intermédiaire de $i$ sd, le courant $i$ sq contrôle le couple.

L'implantation de cette commande nécessite deux blocs :

- un pour le courant $i_{\mathrm{sq}}$ recevant la consigne de couple, en utilisant un correcteur $\left(C_{\mathrm{iq}}\right)$;

- un pour le flux $\phi_{\mathrm{rd}}$ au travers de $i_{\mathrm{sd}}$, en utilisant deux correcteurs ( $C \phi_{\mathrm{rd}}$ et $\left.C_{\mathrm{id}}\right)$.

Ceci conduit à la structure de la figure 17. 


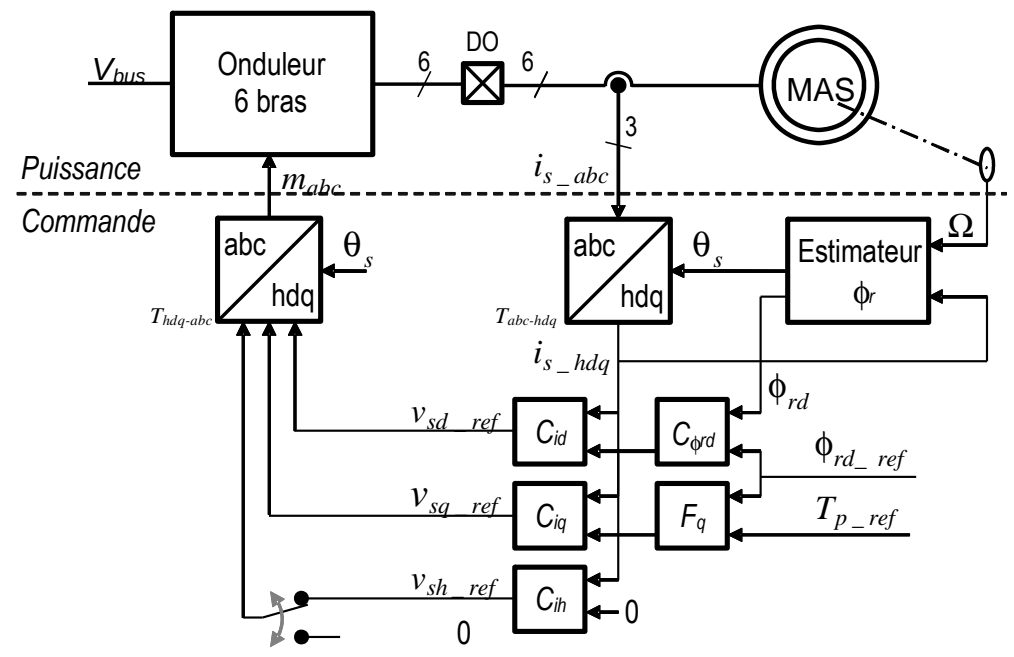

Figure 17. Commande de la MAS pouvant gérer le mode dégradé.

On notera qu'il n'est pas nécessaire de faire un usage généralisé des correcteurs résonants. En effet, le couple dépend du courant $i_{\text {sq }}$ et du flux $\phi_{\mathrm{rd}}$. Ce dernier dépend lui-même du courant $i_{\text {sd. }}$. Pour éliminer des harmoniques à l'origine des ondulations de couple, on ne traite que les courants $i$ sq et $i_{\text {sd }}$ avec des correcteurs $C$ iq et $C$ id de type PI+résonant. Le dernier correcteur $C_{\phi_{\mathrm{rd}}}$ pour le flux $\phi_{\mathrm{rd}}$ n'est donc pas concerné, c'est un PI classique.

Les correcteurs PI+résonant concernés par le mode de réglage spécifique utilisent aussi la marge de stabilité généralisée.

\subsubsection{Analyse des performances en fonctionnement dégradé}

La méthode proposée pour maintenir un couple constant alors qu'une phase d'alimentation est défaillante utilise des correcteurs spécifiques. Pour atteindre notre objectif, les courants dans la machine fictive sont maintenus constants et un courant homopolaire apparaît. La contrepartie d'un couple de même valeur est une augmentation de l'amplitude des courants dans les phases saines conduisant à une augmentation des pertes par effet Joule (Shamsi-Nejad et al., 2008).

\subsubsection{Pertes Joule}

Dans les deux configurations, alimentation saine ou pas, l'obtention d'un couple constant nécessite que la commande impose des courants produisant un champ tournant circulaire. Par conséquent, pour la répartition sinusoïdale des forces 
magnéto-motrices retenue ici, l'état magnétique au rotor de la machine reste le même dans les deux modes de fonctionnement. On réalise alors l'hypothèse que les pertes fer et les pertes Joule rotoriques sont inchangées dans les deux modes de fonctionnement. Par conséquent, la modification des pertes globales dans la machine ne provient que des pertes Joule statoriques $\left(p_{\mathrm{j} s}\right)$. De plus, la transformation en machines fictives (Concordia et Park) utilisée est orthogonale, ce qui garantit la conservation de la norme, et, par conséquent, celle de la puissance, qu'elle soit exprimée dans le repère $a b c$ des grandeurs réelles ou dans le repère $h d q$ des machines fictives. L'expression de la puissance $p_{\mathrm{js}}$ instantanée est alors :

$$
p_{j s}(t)=R_{s}\left[\mathbf{i}_{s a}^{2}(t)+i_{s b}^{2}(t)+i_{s c}^{2}(t)\right]=R_{s}\left[\mathbf{i}_{s h}^{2}(t)+i_{s d}^{2}(t)+i_{s q}^{2}(t)\right]_{[18]}
$$

En fonctionnement normal, le courant homopolaire est nul. Les pertes cuivre dans $\mathrm{M}_{\mathrm{h}}$ le sont donc aussi. On retrouve alors l'expression classique des pertes Joules moyennes sur une période, exprimée avec les valeurs efficaces des courants :

$$
P_{j s n}=\left.\left\langle p_{j s}(t)\right\rangle\right|_{i_{h}=0}=R_{s}\left(\bigcup_{s d}^{2}+I_{s q}^{2}\right){ }_{[19]}
$$

En cas de défaut, le courant homopolaire produit des pertes supplémentaires. Elles sont évaluées à partir de l'expression [13] du courant homopolaire dépendante de $i_{\mathrm{sd}}$ et $i_{\mathrm{sq}}$ qui est introduite dans [18]. La puissance instantanée $p_{\mathrm{ss}}$ devient :

$$
\begin{aligned}
p_{j s}(t)=R_{s}\left[\left(1+2 \cos ^{2}\left(\omega_{t}+\alpha\right)\right) \cdot i_{s d}^{2}\right. & +\left(1+2 \sin ^{2}\left(\omega_{t}+\alpha\right)\right) \cdot i_{s q}^{2} \\
& \left.-\sin \left(2 \omega_{t}+2 \alpha\right) \cdot i_{s d} i_{s q}\right]
\end{aligned}
$$

Où l'on rappelle que l'angle $\alpha$ dépend de la phase ouverte (relation [13]).

Avec les valeurs efficaces des courants, la puissance en dégradé s'exprime par :

$$
P_{j s d}=\left.\left\langle p_{j s}(t)\right\rangle\right|_{i_{h} \neq 0}=2 R_{s}\left(V_{s d}^{2}+I_{s q}^{2}\right)[21]
$$

À partir de cette évaluation, on remarque que les pertes Joule sont doublées par rapport au fonctionnement normal si on conserve les mêmes valeurs de $I$ sd et $I_{\mathrm{sq}}$. À partir de cette analyse, on peut alors envisager deux cas de figure :

- le premier consiste effectivement à garder les mêmes courants fictifs, ce qui permet de produire le même couple qu'en fonctionnement normal, mais en entraînant le doublement des pertes Joule ;

- le second cherche à dissiper les mêmes pertes cuivre statoriques qu'en mode normal $(P$ jsn $)$. Dans ce cas, il est important de préserver la magnétisation de la 
machine fixée pour le fonctionnement normal en ne modifiant pas le courant $i \quad$ sd. Dans ce cas, le courant $I$ 'sq correspondant s'écrit :

$$
I_{s q}^{\prime}=\sqrt{\frac{I_{s q}^{2}-I_{s d}^{2}}{2}}
$$

Dans l'hypothèse d'un comportement linéaire, le rapport des couples est le même que celui des courants et le couple qui subsiste en fonctionnement dégradé s'exprime par :

$$
T^{\prime}=\frac{I_{s q}^{\prime}}{I_{s q}} T=\sqrt{\frac{1}{2}\left(1-\left(\frac{I_{s d}}{I_{s q}}\right)^{2}\right)} T
$$

En conclusion, la stratégie de maintien d'un couple constant dans le cas où deux phases seulement alimentent la machine peut être envisagée en limitant les contraintes thermiques, mais au prix de performances mécaniques moindres. Dans le cas de véhicules électriques par exemple, ce fonctionnement dégradé est envisageable le temps d'atteindre un site de maintenance. Si la performance doit être absolument maintenue avec le même couple, ce sera pendant une courte durée, le temps de terminer l'action en cours (par exemple dans le cas de pompes dans une installation nucléaire).

\subsubsection{Effets sur les courants et les tensions}

Si le couple délivré par la machine à la charge mécanique est maintenu, on envisage deux conséquences principales sur la chaîne électrique d'alimentation :

- la première est un accroissement du courant dans les phases saines qui surcharge les bras correspondants de l'onduleur ;

- la seconde est un accroissement de la tension appliquée aux enroulements, compatible ou pas avec les limites d'isolement, ou pouvant être la cause de la saturation de l'onduleur.

\section{Effets sur les courants}

On cherche ici à évaluer l'amplitude des courants pour s'assurer à la fois de leur valeur et de leur équilibre ou pas dans les phases saines de l'alimentation.

L'augmentation de l'amplitude maximale des courants dans les phases restantes est induite par la stratégie de conservation du couple. À cette condition, il faut ajouter le maintien de la magnétisation de la machine avec le même courant $i$ 
tandis que la conservation du couple impose le même courant $i$ machine principale est dans le même état, c'est sur le courant $i$

sq. Puisque la sh de la machine homopolaire que se reporte la dégradation et l'expression de sa valeur efficace issue de [13] est :

$$
I_{s h}=\sqrt{I_{s q}^{2}+I_{s d}^{2}}
$$

L'évaluation de la valeur efficace des courants réels dans les phases saines passe d'abord par l'évaluation des courants instantanés. Ils sont obtenus en appliquant la transformation $T_{h d q-a b c}$ au vecteur des courants fictifs de composantes $\left(i_{\mathrm{sh}}, i_{\mathrm{sd}}, i_{\mathrm{sq}}\right)$. Le courant homopolaire est éliminé en utilisant la relation [13] et conduit aux expressions des courants réels en mode dégradé :

$$
\begin{aligned}
& i_{s a}=2 \sqrt{\frac{2}{3}}\left(i_{s d} \sin \left(\theta-\frac{\alpha}{2}\right)+i_{s q} \cos \left(\theta-\frac{\alpha}{2}\right)\right) \sin \left(-\frac{\alpha}{2}\right) \\
& i_{s b}=2 \sqrt{\frac{2}{3}}\left(i_{s d} \sin \left(\theta-\frac{\alpha}{2}-\frac{\pi}{3}\right)+i_{s q} \cos \left(\theta-\frac{\alpha}{2}-\frac{\pi}{3}\right)\right) \sin \left(\frac{\pi}{3}-\frac{\alpha}{2}\right) \\
& i_{s c}=2 \sqrt{\frac{2}{3}}\left(i_{s d} \sin \left(\theta-\frac{\alpha}{2}-\frac{2 \pi}{3}\right)+i_{s q} \cos \left(\theta-\frac{\alpha}{2}+\frac{2 \pi}{3}\right)\right) \sin \left(\frac{2 \pi}{3}-\frac{\alpha}{2}\right)
\end{aligned}
$$

Or, avec l'action des correcteurs résonants, $i$ sd et $i$ sq sont constants en régime permanant (notés $I$ sd et $I$ sq), ce qui permet de déterminer que les deux courants efficaces dans les phases saines sont identiques et s'expriment :

$$
I_{s k}=\sqrt{I_{s q}^{2}+I_{s d}^{2}} \text { quelque soit } k=a, b \text { ou } c \text { la phase saine considérée }
$$

En conclusion, les courants parcourant les phases saines sont de même amplitude que le courant homopolaire.

\section{Effets sur les tensions}

Comme pour les courants, on cherche ici à évaluer les tensions appliquées aux phases saines de la machine en cas de dégradation.

La tension statorique homopolaire instantanée est donnée par : 


$$
\begin{aligned}
v_{s h}(t) & =R_{s} i_{s h}(t)+L_{s h} \frac{\mathrm{d} i_{s h}(t)}{\mathrm{d} t} \\
& =\sqrt{2}\left[\mathrm{~d}_{s} \Omega_{s}\right)+L_{s h} \omega I_{s d} \sin \left(\omega_{t}-\alpha\right)+-R_{s} I_{s d}+L_{s h} \omega_{s q} \cos \left(\omega_{t}-\alpha\right)
\end{aligned}
$$

Cette relation permet d'exprimer la tension homopolaire efficace en régime permanent :

$$
V_{s h}=\sqrt{\left(R_{s}^{2}+Q_{s h} \omega^{2}\right) V_{s q}+I_{s d}^{2}}
$$

L’impédance homopolaire $Z_{\text {sh }}$ est ainsi mise en évidence :

$$
Z_{s h}=\sqrt{R_{s}^{2}+()_{s h} \omega^{2}}
$$

Pour poursuivre l'évaluation des tensions de phase, on s'appuie sur les considérations suivantes :

- la correction du couple impose des courants fictifs constants, ce qui est obtenu par l'emploi de correcteurs résonants ;

- les expressions des courants fictifs ont été établies dans différentes stratégies de fonctionnement dégradé aux paragraphes précédents relatifs aux pertes Joule ou aux courants par phase.

Les tensions aux bornes des machines fictives sont calculées en superposant les tensions engendrées par les fonctionnements normal et dégradé, c'est à dire :

- les tensions $v_{\mathrm{sa}}, v_{\mathrm{sb}}$ et $v_{\mathrm{sc}}$ en cas d'alimentation normale ;

- les contributions $v \quad$ sha $\quad v_{\text {shb }}$ et $v$ shc obtenues par la transformation $T \quad h d q-a b c$ appliquée à la tension $v_{\text {sh. }}$.

Si $V$ est la tension efficace commune des tensions $V$ sa, $V_{\text {sb }}$ et $V$ sc avec une alimentation normale, les expressions évoluent avec une alimentation par deux phases et deviennent :

$$
\left(\begin{array}{c}
v_{s a}^{\prime} \\
v_{s b}^{\prime} \\
v_{s c}^{\prime}
\end{array}\right)=T_{h d q-a b c}\left(\begin{array}{c}
v_{s h} \\
0 \\
0
\end{array}\right)+\left(\begin{array}{l}
v_{s a} \\
v_{s b} \\
v_{s c}
\end{array}\right)=\left(\begin{array}{c}
\frac{v_{s h}}{\sqrt{3}}+V \sqrt{2} \sin \left(\omega_{t}\right)_{t} \\
\frac{v_{s h}}{\sqrt{3}}+V \sqrt{2} \sin \left(\omega_{t}-\frac{2 \pi}{3}\right) \\
\frac{v_{s h}}{\sqrt{3}}+V \sqrt{2} \sin \left(\omega_{t}-\frac{4 \pi}{3}\right)
\end{array}\right)
$$


L'expression de $v$ sh tirée de [29] est introduite dans [32] pour aboutir à trois tensions dont les amplitudes maximales sont sensiblement différentes comme l'illustre la figure 18 pour laquelle les tensions saines sont repérées en traits fins (les valeurs numériques sont celles de la machine d'application définie en annexe V).

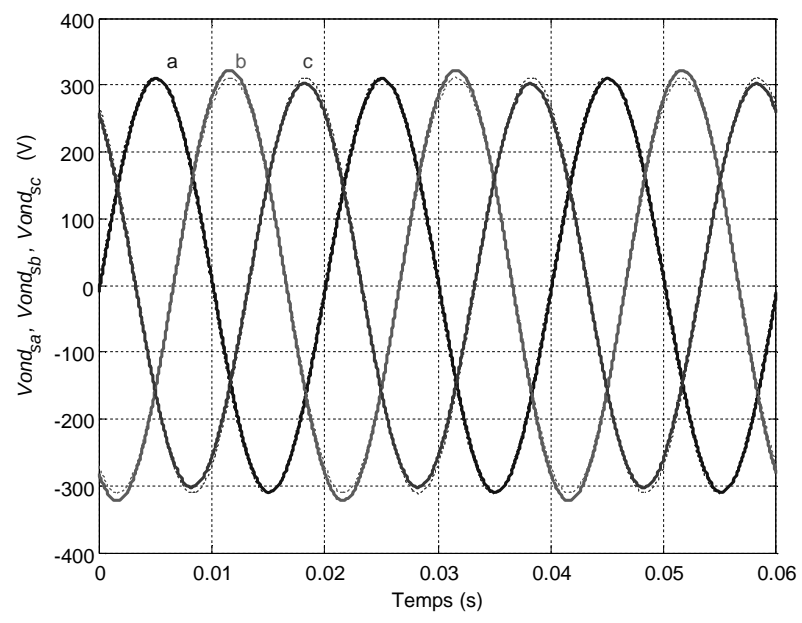

Figure 18. Tensions issues des onduleurs : phases saines (lignes interrompues) et une phase ouverte (lignes pleines).

Pour cette illustration, la phase $b$ est ouverte. Cela correspond à un accroissement de 3,5\% environ de l'amplitude de la tension délivrée par l'onduleur pour cette phase ouverte et une légère diminution pour la phase $\mathrm{c}(2,9 \%$ environ), la dernière étant quasiment inchangée.

Si l'impact d'une dégradation sur l'amplitude des courants sains est particulièrement visible et notable, les variations des tensions statoriques sont moins pénalisantes. Les quelques pourcents de variation de la tension nominale peuvent être tolérées sans craindre la saturation de l'onduleur.

\subsubsection{Vérifications en simulation}

Les simulations sont menées sur une machine asynchrone de puissance comparable à la machine synchrone étudiée au $§ 3.2$ à la vitesse constante de 1500 $\mathrm{tr} / \mathrm{min}$. Les caractéristiques de la machine sont regroupées en annexe V.

Dès l'ouverture d'une phase statorique, les observations effectuées sur la machine avec les correcteurs PI n'offrent pas d'informations supplémentaires par rapport à la machine synchrone : le courant homopolaire fait son apparition et le couple est très fortement pulsé. 
Pour apprécier l'augmentation des grandeurs, la consigne de couple est maintenue en mode dégradé. Le couple varie pendant une courte durée que l'on peut estimer à moins d'une période du réseau (figure 19 gauche). Le retour du couple à la «normale » est confirmé par la forme du courant $i$ sq visible sur la figure 19 droite. On y observe aussi que le courant homopolaire n'est plus nul, tandis que sa période de $20 \mathrm{~ms}$ est celle des grandeurs statoriques.
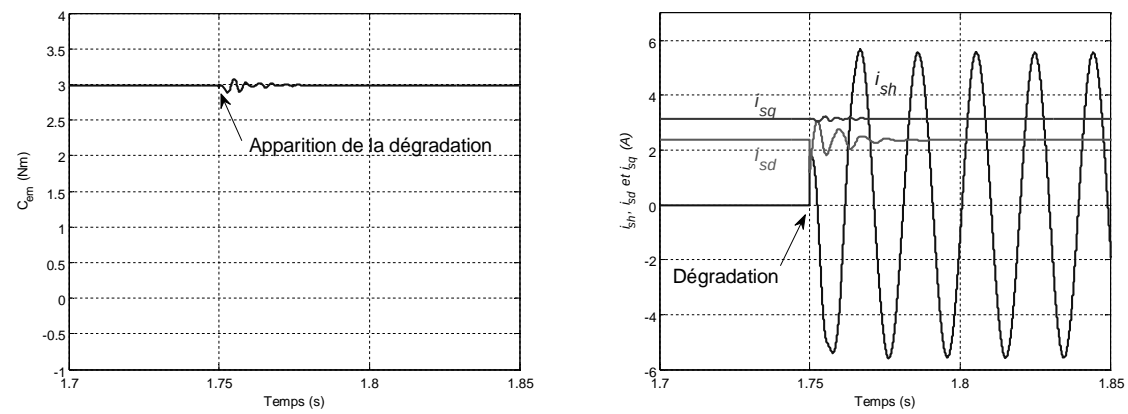

Figure 19. Comportement du couple (à gauche) et courants fictifs (à droite).

Dans les mêmes conditions, on peut apprécier l'augmentation des amplitudes maximales des courants par enroulement alimenté (figure 20 gauche).

L'accroissement d'un facteur 1,7 (proche de $\sqrt{3}$ ), correspond au doublement des pertes par effet Joule. De plus, l'amplitude maximale du courant homopolaire (figure 19 droite) est la même que celle dans les phases saines (environ 5,5 A) comme l'établissent les relations [24] et [28]. Pour parvenir à des courants de même amplitude dans les phases saines, les onduleurs délivrent des tensions non équilibrées à la machine (figure 20 droite), d'amplitude toutes différentes comme ceci est établi avec [32] et illustré sur la figure 18.
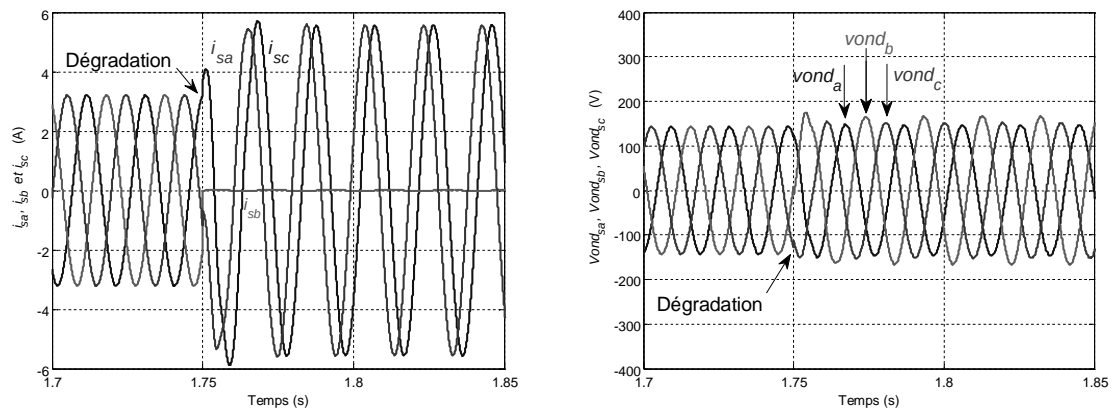

Figure 20. Courants (à gauche) et tensions (à droite) statoriques avant et après l'apparition du défaut. 


\subsubsection{Validation expérimentale}

Les vérifications expérimentales ont été effectuées sur la machine définie en annexe $\mathrm{V}$, en utilisant un banc expérimental DSpace 1005 piloté avec l'environnement de développement temps réel ControlDesk ${ }^{\mathrm{TM}}$ (figure 21 gauche).

La figure 21 droite montre le couple sur l'arbre estimé à partir des courants mesurés dans les phases. En mode dégradé, et avec la correction proposée, il est quasi confondu avec celui en fonctionnement normal. Pour les conditions d'étude, le couple obtenu lorsque seul le correcteur PI de base agit est ondulé comme l'a montré l'étude. Sa période de 13 ms correspond, aux variations de réglage près, à la moitié de la période des grandeurs statoriques, ce qui valide l'existence de l'harmonique 2 de couple.
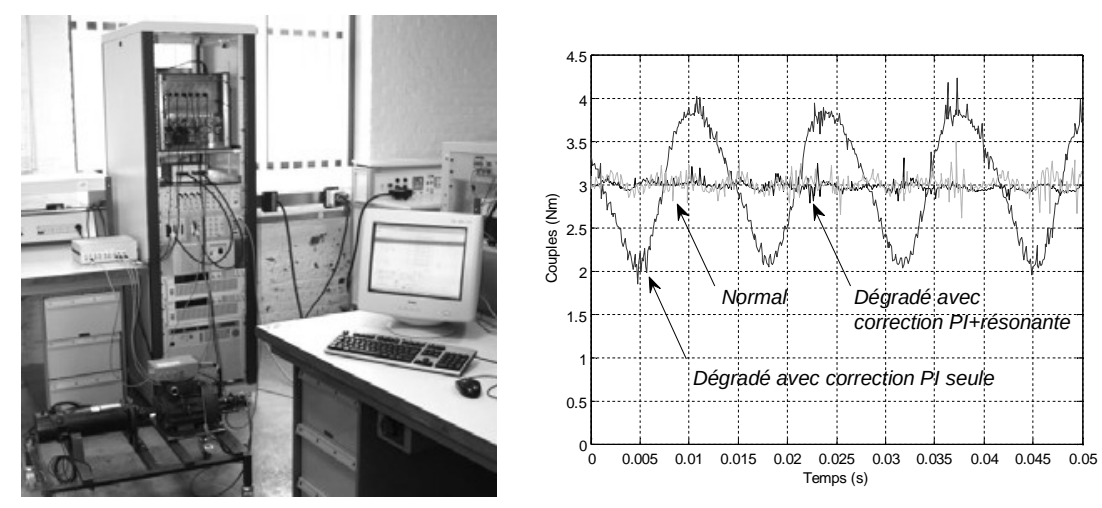

Figure 21. Plateforme expérimentale (gauche). Couples estimés sur l'arbre (droite).
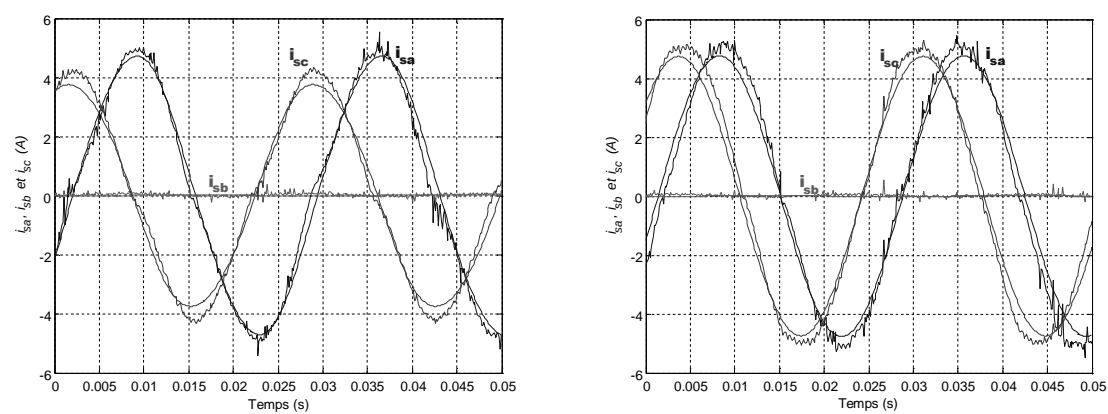

Figure 22. Courants statoriques avec correction PI (gauche) et résonante (droite). 

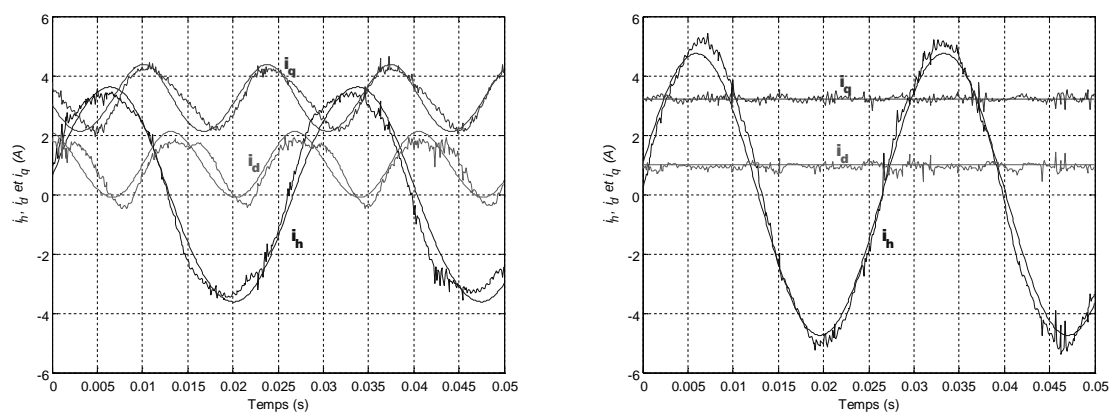

Figure 23. Courants dans Mp avec correction PI (gauche) et résonante (droite).

En choisissant d'ouvrir la phase 2 de l'alimentation de la machine, les figures 22 et 23 montrent la quasi coïncidence des courants issus de la simulation et de l'expérimentation. On trouve à gauche les courants quand les correcteurs PI sont actifs, tandis qu'à droite les correcteurs résonants agissent efficacement. Ces derniers rétablissent des courants d'amplitude maximale identiques dans les phases saines (figures 22), ce qui conduit à des courants constants dans la machine principale. Le courant homopolaire non nul, de fréquence moitié de celle des perturbations, rend compte des effets de la dégradation.

\subsection{Conclusion}

La confrontation des résultats de manipulation à ceux issus de la simulation montre une grande similitude des grandeurs. Ce constat atteste de la validité du modèle de dégradation retenu. La méthode décrite permet de préserver un couple constant sur l'arbre quand l'alimentation statorique est défaillante. La mise en œuvre pratique ne nécessite aucune intervention particulière quand une phase, quelle qu'elle soit, disparaît. En fonction du choix initial opéré pour conserver le couple ou les courants en ligne identiques, la machine délivre toujours le couple constant particulièrement utile dans les exemples énoncés dans l'introduction.

\section{Conclusion générale et perspectives}

Cet article a rappelé que pour les machines triphasées synchrones ou asynchrones, la perte d'une phase d'alimentation amène un couple pulsatoire à la fréquence double du fondamental de celle des grandeurs statoriques. L'originalité de l'analyse et du traitement visant à rendre l'entraînement tolérant à la dégradation de son alimentation électrique réside dans la proposition d'une structure de correction ne nécessitant pas : 
- une modification structurelle de la commande ;

- la connaissance de la phase ouverte.

Pour mener à bien cette solution, une modélisation vectorielle, pouvant s'appliquer à toutes les machines disposant d'un nombre quelconque de phases, est d'abord décrite, puis appliquée à la machine triphasée non couplée (qui est un type élémentaire de machine polyphasée) en utilisant la Représentation Énergétique Macroscopique (REM). La dégradation est ensuite modélisée sur le même principe en s'appuyant sur la « resistor companion method ». La machine et son alimentation ainsi modélisées, la commande de l'entraînement est élaborée, soit par inversion de la REM pour une machine synchrone, soit par adaptation d'une commande industrielle pour une machine asynchrone. L'analyse des degrés de liberté du système dégradé conduit à abandonner le contrôle du courant homopolaire au travers d'une adaptation de la structure de commande établie. Enfin, le manque d'efficacité de simples correcteurs proportionnel et intégral pour rejeter les perturbations conduit à analyser le contenu harmonique des grandeurs à l'origine du couple. L'amélioration consiste alors à ajouter des correcteurs résonants accordés sur la fréquence des perturbations pour éliminer efficacement les ondulations de couple.

L'évaluation des pertes Joule, des courants et des tensions statoriques permet d'apprécier les effets de la dégradation sur les performances de la machine. De plus, la modification des grandeurs en fonctionnement dégradé met en évidence l'impact du défaut sur la source d'alimentation.

Les analyses en simulation valident l'efficacité de la modélisation et de la méthode de traitement de la dégradation proposée, analyses confirmées par les résultats expérimentaux.

Finalement, il est à noter que les modélisations ont été menées en utilisant le formalisme vectoriel et la représentation énergétique macroscopique. Même si les machines d'application sont triphasées, la méthode peut être directement étendue à des machines polyphasées disposant de plus de trois phases. Dans celles-ci, plus de phases peuvent être ouvertes (même si la probabilité d'une telle situation diminue avec le nombre de phases de la machine). Le plus grand nombre de degrés de liberté permet une plus grande aisance dans la mise en œuvre d'une stratégie de correction du couple en adaptant les correcteurs des boucles de courant. Ces correcteurs prendront en compte la composition harmonique des courants dans les machines fictives qu'ils rejetteront pour lisser le couple restant. Dans ce cadre, on peut envisager que l'entraînement poursuivra son fonctionnement pour peu que perdurent deux phases au minimum. Mais ce régime ne sera possible qu'en admettant des performances de l'entraînement fortement dégradées. 


\section{Remerciements}

Les auteurs tiennent à remercier Fabrice Locment pour son concours dans la validation expérimentale du correcteur spécifique PI+résonant .

\section{Bibliographie}

Barre P. J., Bouscayrol A., Delarue P., Dumetz E., Giraud F., Hautier J. P., Kestelyn X., Lemaire-Semail B., Semail E., «Inversion-based control of electromechanical systems using causal graphical descriptions », IECON'2006, Paris, November 6-10, 2006, ISBN $1-4244-0136-4$

Baumann C., Piquet H., Roboam X., «A novel structure for aeronautic DC distribution: the Mixed function for Actuation \& Power Flow Control (MAPFC) », International Electric Machines and Drives Conference (IEMDC), 2007.

Bennett J.W., Jack A.G., Mecrow B.C., Atkinson D.J., Sewell C., Mason G., «Fault-tolerant control architecture for an electrical actuator », 2004, 35th Annual IEEE Power Electronics Specialists Conference, June 20-25, 2004, Aachen, Germany.

Boldea I. : Nasar S.A., Vector Control of AC Drives, RCR press, 1992.

Bouscayrol, A.; Davat, B.; De Fornel, B.; Francois, B.; Hautier, J.P.; Meibody-TabaR, F.; Pietrzak-David, M.; Multi-machine multi-converter system for drives: analysis of coupling by a global modeling, Industry Applications Conference, 2000, volume 3, 8-12 Oct. 2000, Page(s): 1474 - 1481 vol.3.

Bouscayrol, A., Formalisme de représentation et de commande appliqués aux systèmes électromécaniques multimachines multiconvertisseurs, Mémoire d'habilitation à diriger des recherches soutenue le 22 décembre 2003, Université des Sciences et Techniques de Lille.

Caron J.P., Hautier J.P., Modélisation et commande de la machine asynchrone , Éditions Technip - Electrotechnique - Vol. 7 - 280 pages, Paris, 1995.

Crévits Y., Kestelyn, X., Semail, E., « Investigation on vector control of three-phase synchronous machines under supply fault condition », International Symposium on Power Electronics, Electrical Drives, Automation and Motion (SPEEDAM). May 2006, pp. 439444.

De Fornel B. (sous la direction de J.-P. Louis), Modèles pour la commande des actionneurs électriques, partie 5 : «modélisation dynamique des machines asynchrones », traité EGEM série Génie électrique, ISBN 2-7462-0917-9, Hermès, Lavoisier, 2004, pp. 215287.

Ertugrul N., Soong W., Dostal G., Saxon D., « Fault tolerant motor drive system with redundancy for critical applications », Conf. Rec. IEEE Power Electronics Specialists Conf., vol. 3, 2002, pp. 1457-1462.

Figueroa J., Cros J., Viarouge P., « Polyphase PM brushless DC motor for high reliability application », Proc. of EPE 2003, Toulouse (France), Sept.2003, CD-ROM. 
Figueroa J., Cros J., Viarouge P., « Generalized transformations for polyphase phaseModulation motors », IEEE Transaction on Energy Conversion, Vol. 21, Issue 2, pp. 332 341, June 2006, ISSN: 0885-8969.

Filliau G., Alain Bondu A., Mazodier L., «Le navire tout électrique - Évolutions et systèmes de conduite », traité les techniques de l'ingénieur, réf. D5620.

Fu J.R., Lipo T.A., « Disturbance-Free Operation of a Multiphase Current-Regulated Motor Drive with an Open Phase », IEEE Transactions on Industry Applications, Vol. 30, no 5, September/October 1994, pp. 1267-1274.

Jahns T.M.. «Improved reliability in solid-state ac drives by means of multiple independent phase-drive units », IEEE Transactions on Industry Applications, Vol. IA16, pp. 321-331, May/June 1980.

Kestelyn X., Modélisation vectorielle multimachines pour la commande des ensembles convertisseurs-machines polyphasés, thèse de Doctorat soutenue le 4 décembre 2003, Université des Sciences et Techniques de Lille.

Kestelyn X., Locment F., Crévits Y. and Semail E., « Easy-to-implement integral numerical simulation of multi-phase drives under fault supply condition » », International Electric Machines and Drives Conference (IEMDC), 5-2007.

Klingshirn E.A., «High phase order induction motors - Part 1 - Description and theorycal considerations and Part 2 - Experimental results », IEEE Transaction On Power Apparatus and Systems, Vol. PAS-102, No 1, January 1983.

Klingshirn E.A., « Harmonic filters for six-phase and other multiphase motors on voltage source inverters », IEEE Transaction On Industry Applycation ，Vol. IA-21, No 4, may/june 1985 .

Levi, E.; Bojoi, R.; Profumo, F.; Toliyat, H.A.; Williamson, S., « Multiphase induction motor drives - A technology status review », Electric Power Applications, IET Volume 1, Issue 4, July 2007, Page(s) : $489-516$.

Lhomme W., Gestion d'énergie dans un véhicule hybride basé sur la représentation énergétique macroscopique, thèse de Doctorat soutenue le 16 novembre 2007, Université des Sciences et Techniques de Lille.

Locment F., Semail E., Kestelyn X., «A vector controlled axial-flux seven-phase machine in fault operation », Proc. Int. Conf. on Electrical Machines ICEM, Chania, Crete, Greece, 2006, CD-ROM paper.

Martin J.P., Meibody-Tabar F., Davat B., « Multiple-phase Permanet Magnet Synchronous Machine Supplied By VSIs, Working under Fault Conditions », IEEE Industry Applications Society, 2000, CD ROM.

Martin J.P., Pierfederici S., Meibody-Tabar F., Letellier P., « Synthèse des méthodes de filtrage du Couple des MSAP Polyphasées en modes normal et dégradé », Revue Internationale de Génie Électrique, vol. 10/1-2-2007, pp. 117-149.

Monti A., Santi E., Dougal R., Ponci F., Riva M., «Symbolic analysis for automatic model generation 2001 ». IEEE Power Engineering Society Summer Meeting , Vol. 3, pp. 14451450, 15-19 July 2001. 
Ryu H.M., Kim J. H., Sul S. K., « Synchronous Frame Current Control of Multi-Phase Synchronous Motor, Part I. Modeling and Current Control Based on Multiple d-q Spaces Concept Under Balanced Condition », Proc. of IEEE-IAS'04, Vol. 1, pp. 56-63, Seattle (USA), Oct. 2004.

Ryu H.M., Kim J. H., Sul S. K., "Synchronous Frame Current Control of Multi-Phase Synchronous Motor, Part II. Asymmetric Fault Condition due to Open Phases", Proc. of IEEEIAS'04, vol. 1, pp. 268-275, Seattle (USA), Oct. 2004.

Semail E., Outils et méthodologie d'étude des systèmes électriques polyphasés.

Généralisation de la méthode des vecteurs d'espace, thèse de Doctorat soutenue le 30 juin 2000, Université des Sciences et Techniques de Lille.

Semail E., Bouscayrol A., Hautier J.P. «Vectorial formalism for analysis and design of polyphase synchronous machines », European Physical Journal-Applied Physics, Vol. 22 $\mathrm{n}^{\circ} 3,2003, \mathrm{pp} 207-221$.

Semail E., Louis J.-P., Feld G., Modélisation des machines électriques en vue de leur commande-concepts généraux , partie 4 : «propriétés vectorielles des systèmes électriques triphasés », traité EGEM série Génie électrique, ISBN 2-7462-0916-0, Hermès, Lavoisier, 2004, pp. 181-246.

Semail E., Meibody-Tabar F., Benkhoris M. F., Razik H., Pietrzak-David M., Monmasson E., Bouscayrol A., Davat B., Delarue Ph., de Fornel B., Hautier J. P., Louis J. P., Piefederici S., « Représentations SMM de machines polyphasées", RIGE, Revue Internationale de Génie Electrique, vol. 8 n²/2005, pp. 221-239.

Semail E., Kestelyn X., Locment F., «Fault tolerant multiphase electrical drives: the impact of design », IET Colloquium on Reliability in Electromagnetic Systems, Paris (France), May 2007.

Shamsi-Nejad M.A., Nahid-Mobarakeh B., Pierfederici S., Meibody-Tabar F., « Fault Tolerant and Minimum Loss Control of Double-Star Synchronous Machines Under Open Phase Conditions », IEEE TRANSACTIONS ON INDUSTRIAL ELECTRONICS, VOL. 55, NO. 5, MAY 2008.

Toliyat H.A., Lipo T.A., White J.C., « Analysis of a Concentrated Winding Induction Machine for Adjustable Speed Drive Applications Part 1 (Motor Analysis) », Transactions on Energy Conversion, Vol. 6, No.4, December 1991.

Toliyat H.A., Lipo T.A., White J.C., « Analysis of a Concentrated Winding Induction Machine for Adjustable Speed Drive Applications Part 1 and 2 », IEEE Transactions on Energy Conversion, Vol. 6, No.4, December 1991.

Toliyat H.A., « Analysis and Simulation of Five-Phase Variable -Speed Induction Motor Drives Under Asymmetrical Connections », IEEE Transactions on Power Electronics , vol 13 n4, pp. 748-756, 1998.

Wallmark O., Harnefors L., Carlson O., « Control Algorithms for a Fault-Tolerant PMSM Drive », IEEE TRANSACTIONS ON INDUSTRIAL ELECTRONICS, VOL. 54, NO. 4, AUGUST 2007. 
Welchko B. A., Jahns T. M. and Hiti S., « IPM synchronous machine drive response to a single-phase open circuit fault », IEEE Transaction on power electronics , September 2002 , volume 17 , issue 5 .

Welchko B.A., Lipo T.A., Jahns T.M., Schulz S.E., «Fault tolerant three-phase AC motor drive topologies: a comparison of features, cost, and limitations », IEEE Transactions on Power Electronics, July 2004, volume 19, issue 4.

Williamson S., Smith S., Hodge C., « Fault tolerance in multiphase propulsion motors », Journal of Marine Engineering and Technology, n4, A4, 2004.

Wulveryck Marc, Contrôle de courants alternatifs par correcteur résonant multifréquentiel Application à la commande de systèmes électrotechniques non linéaires, thèse soutenue à l'université de Lille 1, 2000, No : 00 LIL1 0062

Zheng J., Guillaud X., Degobert P., « Control of AC machines with Multi-Frequency Resonant Controller », proceeding of the 11th International Power Electronics and Motion Control Conference (EPE-PEMC), September 2004, Riga, Latvia.

Zhao Y, Lipo T.A., « Space Vector PWM Control of Dual Three-phase Induction Machine Using Vector Space Decomposition », IEEE Transactions On Industry Applications , VOL. 31, NO. 5, September-October 1995, pp. 1100-1109.

Zhao Y., Lipo T.A., « Modeling and control of a multi-phase induction machine with structural unbalance. Part I: Machine modeling and multi-dimensional current regulation », IEEE Transactions on Energy Conversion , vol. 11, no. 3, September 1996, pp. 570-577.

Zhao Y., Lipo T.A., « Modeling and control of a multi-phase induction machine with structural unbalance. Part II: Field-oriented control and experimental verification », IEEE Transactions on Energy Conversion, vol. 11, no. 3, September 1996, pp. 578-584. 


\section{Annexes}

Annexe I : relations de la MAS dans le repère de Park conservant la puissance

Relations au stator (indices s)

\begin{tabular}{|c|c|c|c|}
\hline Axes & & Relations & \\
\hline \multirow{2}{*}{ Tensions } & $\mathrm{d}$ & $v_{s d}=R_{s} \cdot i_{s d}+{ }_{s} \cdot \phi_{s d}-\omega_{s} \cdot \phi_{s q}$ & {$[\mathrm{~A} 1-1]$} \\
\hline & $\mathrm{q}$ & $v_{s q}=R_{s} \cdot i_{s q}+{ }_{s} \cdot \phi_{s q}+\omega_{s} \cdot \phi_{s d}$ & [A1-2] \\
\hline \multirow{2}{*}{ Flux } & $\mathrm{d}$ & $\phi_{s d}=L_{s} \cdot i_{s d}+M \cdot i_{r d}$ & {$[\mathrm{~A} 1-3]$} \\
\hline & $\mathrm{q}$ & $\phi_{s q}=L_{s} \cdot i_{s q}+M \cdot i_{r q}$ & [A1-4] \\
\hline
\end{tabular}

Relations au rotor (indices $\mathrm{r}$ )

\begin{tabular}{|c|c|c|c|}
\hline Axes & & Relations & \\
\hline \multirow{2}{*}{ Tensions } & $\mathrm{d}$ & $v_{r d}=R_{r} \cdot i_{r d}+{ }_{s} \cdot \phi_{r d}-\omega_{r} \cdot \phi_{r q}$ & {$[\mathrm{~A} 1-5]$} \\
\hline & $\mathrm{q}$ & $v_{r q}=R_{r} \cdot i_{r q}+{ }_{s} \cdot \phi_{r q}+\omega_{r} \cdot \phi_{r d}$ & [A1-6] \\
\hline \multirow{2}{*}{ Flux } & d & $\phi_{r d}=L_{r} \cdot i_{r d}+M \cdot i_{s d}$ & [A1-7] \\
\hline & $\mathrm{q}$ & $\phi_{r q}=L_{r} \cdot i_{r q}+M \cdot i_{s q}$ & {$[\mathrm{~A} 1-8]$} \\
\hline
\end{tabular}

Annexe II : expressions des f.e.m. et des courants apparaissant nécessaires à la construction la REM de la machine asynchrone

Expressions des f.e.m.

\begin{tabular}{|l|l|ll|}
\cline { 2 - 5 } Rotor & & Relations & \\
\hline \multirow{3}{*}{ Stator } & $\mathrm{d}$ & $e_{s d}=-\frac{M}{\tau_{r}} i_{r d}-\omega_{s} \cdot \phi_{s q}+\omega_{r} \frac{M}{L_{r}} \phi_{r q}$ & [A2-1] \\
\cline { 2 - 5 } & $\mathrm{q}$ & $e_{s q}=-\frac{M}{\tau_{r}} i_{r q}+\omega_{s} \cdot \phi_{s d}-\omega_{r} \frac{M}{L_{r}} \cdot \phi_{r d}$ & [A2-2] \\
\cline { 2 - 5 } & $\mathrm{d}$ & $e_{r d}=-\frac{M}{\tau_{s}} i_{s d}+\omega_{s} \frac{M}{L_{s}} \phi_{s q}-\omega_{r} \phi_{r q}$ & [A2-3] \\
\hline
\end{tabular}


Expressions des courants fictifs

\begin{tabular}{|l|c|lc|}
\cline { 2 - 4 } \multicolumn{1}{c|}{ Axes } & & Relations \\
\hline \multirow{3}{*}{ Rotor } & $\mathrm{d}$ & $i_{s d}=\frac{1}{R_{s}} \frac{1}{1+\sigma \tau_{s} s}\left(v_{s d}-\frac{M}{L_{r}} v_{r d}-e_{s d}\right)$ & [A2-5] \\
\cline { 2 - 4 } & $\mathrm{q}$ & $i_{s q}=\frac{1}{R_{s}} \frac{1}{1+\sigma \tau_{s} s}\left(v_{s q}-\frac{M}{L_{r}} v_{r q}-e_{s q}\right)$ & [A2-6] \\
\hline \multirow{3}{*}{ Stator } & $\mathrm{d}$ & $i_{r d}=\frac{1}{R_{r}} \frac{1}{1+\sigma \tau_{r} s}\left(v_{r d}-\frac{M}{L_{s}} v_{s d}-e_{r d}\right)$ & [A2-7] \\
\cline { 2 - 4 } & $\mathrm{q}$ & $i_{r q}=\frac{1}{R_{r}} \frac{1}{1+\sigma \tau_{r} s}\left(v_{r q}-\frac{M}{L_{s}} v_{s q}-e_{r q}\right)$ & [A2-8] \\
\hline
\end{tabular}

Avec $\sigma$ le coefficient de dispersion de Blondel : $\sigma=1-\frac{M^{2}}{L_{r} L_{s}}$.

Annexe III : expressions des grandeurs mécaniques dans la machine asynchrone

Relation liant les pulsations et la vitesse rotorique

$$
\omega_{s}=p \Omega+\omega_{r}[\mathrm{~A} 3-1]
$$

Expressions du couple électromagnétique dans la machine principale

$$
\begin{aligned}
& C_{p}=p \frac{M}{L_{r}}(\phi)_{r d} \cdot i_{s q}-\phi_{r q} \cdot i_{s d} \quad[\mathrm{~A} 3-2] \\
& C_{p}={ }_{p}\left(\phi_{s d} \cdot i_{s q}-\phi_{s q} \cdot i_{s d}\right)_{[\mathrm{A} 3-3]} \\
& C_{p}=p \cdot M\left(i_{s q} \cdot i_{r d}-i_{s d} \cdot i_{r q}\right)_{[\mathrm{A} 3-4]}
\end{aligned}
$$


Annexe IV : expressions des termes de la matrice des couplages fictifs

La matrice couplant les grandeurs dans les machines fictives s'écrit :

$$
\begin{aligned}
R_{F 3 \_} h d q & =\left[\begin{array}{lll}
R_{F 11} & R_{F 12} & R_{F 13} \\
R_{F 21} & R_{F 22} & R_{F 23} \\
R_{F 31} & R_{F 32} & R_{F 33}
\end{array}\right] \\
R_{\text {DO3-hdq }}= & {\left[\begin{array}{lll}
R_{\text {Oh }} & R_{\text {Ohd }} & R_{\text {Ohq }} \\
R_{\text {Ohd }} & R_{\text {Od }} & R_{\text {Odq }} \\
R_{\text {Ohq }} & R_{\text {Odq }} & R_{\text {Oq }}
\end{array}\right] \cdot\left[\begin{array}{l}
i_{h} \\
i_{d} \\
i_{q}
\end{array}\right][\mathrm{A} 4-1] }
\end{aligned}
$$

Avec les résistances $R_{\mathrm{a}}, R_{\mathrm{b}}$ et $R_{\mathrm{c}}$ des dipôles ouvrants dans les phases $a, b$ et $c$, les résistances fictives s'expriment par:

$$
\begin{aligned}
& R_{O h}=\frac{1}{3}\left(R_{a}+R_{b}+R_{c}\right) \\
& R_{O d}=\frac{1}{3}\left(R_{a}+R_{b}+R_{c}\right)+\frac{1}{6}\left(2 R_{a}-R_{b}-R_{c}\right) \cos \left(2 p^{\theta}\right)-\frac{\sqrt{3}}{6}\left(R_{b}-R_{c}\right) \sin (2 p \theta) \\
& R_{O q}=\frac{1}{3}\left(R_{a}+R_{b}+R_{c}\right)-\frac{1}{6}\left(2 R_{a}-R_{b}-R_{c}\right) \cos (2 p \theta)-\frac{\sqrt{3}}{6}\left(R_{b}-R_{c}\right) \sin (2 p \theta) \\
& R_{O h d}=\frac{\sqrt{2}}{6}\left(2 R_{a}-R_{b}-R_{c}\right) \cos \left(p^{\theta}\right)+\frac{\sqrt{6}}{6}\left(R_{b}-R_{c}\right) \sin (p \theta) \\
& R_{O h q}=-\frac{\sqrt{2}}{6}\left(2 R_{a}-R_{b}-R_{c}\right) \sin (p \theta)+\frac{\sqrt{6}}{6}\left(R_{b}-R_{c}\right) \cos (p \theta) \\
& R_{O d q}=-\frac{1}{6}\left(2 R_{a}-R_{b}-R_{c}\right) \sin \left(2 p^{\theta}\right)-\frac{\sqrt{3}}{6}\left(R_{b}-R_{c}\right) \cos (2 p \theta)
\end{aligned}
$$

\section{Annexe V : caractéristiques de la machine asynchrone d'application}

Puissance nominale : $P_{\mathrm{n}}=1,5 \mathrm{~kW} ; 2$ paires de pôles ; fréquence nominale statorique : $50 \mathrm{ou} 60 \mathrm{~Hz}$ (essais à $50 \mathrm{~Hz}$ ) ; fréquence de rotation nominale : $1480 \mathrm{tr} / \mathrm{min}$; tensions statoriques nominales $230 / 400 \mathrm{~V}$.

Stator : $R_{\mathrm{s}}=1,97 \Omega ; L_{\mathrm{sf}}=9,8 \mathrm{mH}$.

Rotor : $R_{\mathrm{r}}=1,55 \Omega ; L_{\mathrm{r}}=10,3 \mathrm{mH}$; inductance mutuelle $M_{S R}=210,5 \mathrm{mH}$. 